\title{
A Computational Analysis of Lower Bounds for Big Bucket Production Planning Problems
}

\author{
Kerem Akartunalı • Andrew J. Miller
}

the date of receipt and acceptance should be inserted later

\begin{abstract}
In this paper, we analyze a variety of approaches to obtain lower bounds for multi-level production planning problems with big bucket capacities, i.e., problems in which multiple items compete for the same resources. We give an extensive survey of both known and new methods, and also establish relationships between some of these methods that, to our knowledge, have not been presented before. As will be highlighted, understanding the substructures of difficult problems provide crucial insights on why these problems are hard to solve, and this is addressed by a thorough analysis in the paper. We conclude with computational results on a variety of widely used test sets, and a discussion of future research.
\end{abstract}

Keywords Production Planning · Lot-Sizing · Integer Programming · Strong Formulations · Lagrangian Relaxation

Mathematics Subject Classification (2000) 90C11

\section{Introduction}

Production planning problems have drawn considerable interest from both researchers and practitioners since the seminal paper of Wagner and Whitin [48]. These problems search for the production plan with the minimum total cost (fixed charges such as setup costs and linear charges such as inventory holding

The research carried out was supported in part by the National Science Foundation grant No. DMI 0323299.

Dept. of Management Science, University of Strathclyde 40 George Street, Glasgow G1 1QE, United Kingdom E-mail: kerem.akartunali@strath.ac.uk

IMB, Université de Bordeaux 1 \& RealOpt, INRIA Bordeaux Sud-Ouest 351 Cours de la Libération, 33405 Talence, France

E-mail: andrew.miller@math.u-bordeaux1.fr 
costs) that satisfies customer demand and follows restrictions of the production environment such as those imposed by capacities. The focus of this paper is on multi-level, multi-item production planning problems with "big bucket" capacities, i.e., each resource is shared by multiple items and different items can be produced in a specific time period. These real-world problems are complicated and computationally challenging to solve, often having complicated BOM (Bills of Materials) structures, where the BOM details which items are required to produce each item. The BOM often has multiple levels, where the last level can be thought of as final products, the previous level can be thought of as components required to make final products, and so forth.

Let $N T, N I$ and $N K$ be the number of periods, items, and machine types, respectively. We assume that each machine type operates only on one level, and each level can employ a number of machine types. Note that if a component appears in two or more levels, then it is assumed to be a different item in each different level. The set endp indicates all end-items, i.e. items with external demand; the other items are assumed to have only internal demand. (No generality lost, since any item that has both internal and external demand can be modeled as two distinct items that share a setup variable.) Let $x_{t}^{i}, y_{t}^{i}$, and $s_{t}^{i}$ represent production, setup, and inventory variables for item $i$ in period $t$, respectively. The setup and inventory cost coefficients are indicated by $f_{t}^{i}$ and $h_{t}^{i}$ for each period $t$ and item $i$. Note that production costs might be also included in the problem in a similar fashion to inventory holding costs. The parameter $\delta(i)$ represents the set of immediate successors of item $i$, and the parameter $r^{i j}$ represents the number of items required of $i$ to produce one unit of $j$. Note that $r^{i j}$ is defined not only for immediate dependencies, but for all dependencies between items $i$ and $j$. The parameter $d_{t}^{i}$ is the demand for end-product $i$ in period $t$, and $d_{t, t^{\prime}}^{i}$ is the total demand between $t$ and $t^{\prime}$, i.e.,

$d_{t, t^{\prime}}^{i}=\sum_{\bar{t}=t}^{t^{\prime}} d_{\bar{t}}$. The parameter $a_{k}^{i}$ represents the time necessary to produce one unit of $i$ on machine $k$, and $S T_{k}^{i}$ is the setup time for item $i$ on machine $k$, which has a capacity of $C_{t}^{k}$ in period $t$. Note that each item is processed by a preassigned machine, and we assume that each item is assigned only to one machine (hence, for an item $i^{\prime}$ that is not processed on a machine $k^{\prime}, a_{k^{\prime}}^{i^{\prime}}=0$ and $\left.S T_{k^{\prime}}^{i^{\prime}}=0\right)$. Let $M_{t}^{i}$ be a big number. Then the formulation of the basic 
model follows:

$$
\begin{aligned}
& \min \sum_{t=1}^{N T} \sum_{i=1}^{N I} f_{t}^{i} y_{t}^{i}+\sum_{t=1}^{N T} \sum_{i=1}^{N I} h_{t}^{i} s_{t}^{i} \\
& \text { s.t. } x_{t}^{i}+s_{t-1}^{i}-s_{t}^{i}=d_{t}^{i} \quad t \in[1, N T], i \in e n d p \\
& x_{t}^{i}+s_{t-1}^{i}-s_{t}^{i}=\sum_{j \in \delta(i)} r^{i j} x_{t}^{j} \quad t \in[1, N T], i \in[1, N I] \backslash e n d p \\
& \sum_{i=1}^{N I}\left(a_{k}^{i} x_{t}^{i}+S T_{k}^{i} y_{t}^{i}\right) \leq C_{t}^{k} \quad t \in[1, N T], k \in[1, N K] \\
& x_{t}^{i} \leq M_{t}^{i} y_{t}^{i} \quad t \in[1, N T], i \in[1, N I] \\
& y \in\{0,1\}^{N T \times N I} \\
& x \geq 0 \\
& s \geq 0
\end{aligned}
$$

The constraints (2) and (3) ensure production balance and demand satisfaction for end-items and intermediate items respectively. Note that for the simplicity of the formulation, we assume lead times to be zero (this does not lose generality; if lead times $\Delta^{i}$ for an item $i$ exist, then this can be be introduced in these constraints simply by replacing $x_{t}^{i}$ variables with $x_{t-\Delta^{i}}^{i}$.). W.l.o.g., we also assume the initial inventories to be zero. The constraints (4) are the big bucket capacity constraints, (5) ensure that the setup variable is set to be 1 if there is positive production, and finally (6), (7), and (8) provide the integrality and nonnegativity requirements. Note that we can define $M_{t}^{i}$ as follows, where $k \in[1, N K]$ such that $a_{k}^{i} \neq 0$ :

$$
\begin{array}{ll}
M_{t}^{i}=\min \left(d_{t, N T}^{i}, \frac{C_{t}^{k}-S T_{k}^{i}}{a_{k}^{i}}\right) & i \in \operatorname{endp} \\
M_{t}^{i}=\min \left(\sum_{j \in e n d p} r^{i j} d_{t, N T}^{j}, \frac{C_{t}^{k}-S T_{k}^{i}}{a_{k}^{i}}\right) & i \in[1, N I] \backslash \text { endp }
\end{array}
$$

We next define an echelon reformulation of the problem, see e.g. Pochet and Wolsey [38]. Our motivation for defining this reformulation is that it clearly exhibits the single-item structure that is present for each item, and it therefore enables us to apply results for single-item models to the multi-level model. We first define echelon demand parameters $D_{t}^{i}$ and echelon stock variables $E_{t}^{i}$ :

$$
\begin{array}{ll}
D_{t}^{i}=d_{t}^{i}+\sum_{j \in \delta(i)} r^{i j} D_{t}^{j} & t \in[1, N T], i \in[1, N I] \\
E_{t}^{i}=s_{t}^{i}+\sum_{j \in \delta(i)} r^{i j} E_{t}^{j} & t \in[1, N T], i \in[1, N I]
\end{array}
$$

Note that for (9) to be well-defined, we let $d_{t}^{i}=0$ for all $i \in[1, N I] \backslash e n d p$. Substituting (10) into (2) and (3) for $s_{t}^{i}$, and using the definition (9), we obtain 
an equation that can replace (2) and (3) in the original formulation:

$$
x_{t}^{i}+E_{t-1}^{i}-E_{t}^{i}=D_{t}^{i} \quad t \in[1, N T], i \in[1, N I]
$$

To satisfy (8), we add the following constraints:

$$
\begin{aligned}
& E_{t}^{i} \geq \sum_{j \in \delta(i)} r^{i j} E_{t}^{j} \quad t \in[1, N T], i \in[1, N I] \\
& E \geq 0
\end{aligned}
$$

Finally, to eliminate the inventory variable $s$, we define echelon inventory holding cost $H_{t}^{j}=h_{t}^{j}-\sum_{i=1}^{N I} r^{i j} h_{t}^{i}$ and replace the objective function (1) with

$$
\sum_{t=1}^{N T} \sum_{i=1}^{N I} f_{t}^{i} y_{t}^{i}+\sum_{t=1}^{N T} \sum_{i=1}^{N I} H_{t}^{i} E_{t}^{i}
$$

We can therefore define the feasible region of the production planning problem as $X=\{(x, y, E) \mid(4)-(7),(11)-(13)\}$, which will be referred in the remainder of the paper as the "basic formulation". The production planning problem can be defined as $Z=\min \{(14) \mid(x, y, E) \in X\}$. We could easily include overtime (i.e., extra capacity that can be bought with an additional cost) or backlogging (i.e., satisfying demand later than requested by the customer with a cost for customer dissatisfaction) variables to generalize this basic model, and some of the test problems we consider in Section 4 incorporate them.

For simplicity, we will sometimes use $\operatorname{conv}(a)$ to denote $\operatorname{conv}((x, y, E) \mid(a))$, where $(a)$ is a set of constraints. For example, $\{(x, y) \mid(7) \cap \operatorname{conv}((6))\}$ represents $\{(x, y) \mid(7)\} \cap \operatorname{conv}(\{(x, y) \mid(6)\})$ in our notation.

\subsection{Literature Review}

Even the capacitated version of the single-item production planning problem is $\mathcal{N} \mathcal{P}$-hard (Florian et al. [19] and Bitran and Yanasse [11]) and therefore dynamic programming algorithms are only limited to some special cases, see e.g. Zangwill [51], Florian and Klein [18], Federgruen and Tzur [16]. Therefore, heuristic algorithms have been employed by many researchers with the hope of obtaining good solutions in acceptable computational times. Heuristic frameworks in general use some decomposition ideas, such as Lagrangian-based decomposition (e.g. Trigeiro et al. [46], Tempelmeier and Derstroff [44]), forward scheme and relax-and-fix (e.g. Belvaux and Wolsey [7], Stadtler [43], Federgruen et al. [17], Akartunalı and Miller [1]) and coefficient modification (e.g. Katok et al. [22], Van Vyve and Pochet [47]). The main disadvantages of the heuristic algorithms (unless based on exact methods such as Lagrangian relaxation) are the lack of solution quality guarantee and the lack of useful insights about basic problem structures. 
Mathematical programming results on production planning problems have usually focused on special cases such as single-item problems, and they have been limited for problems with big bucket capacities. We will briefly discuss these techniques in two subgroups: 1) Valid inequalities that are added into the original formulation using separation algorithms, and 2) Extended reformulations that solve the problem in a different variable space.

An early polyhedral study that defines problem-specific valid inequalities for production planning problems is the study of Barany et al. [5], which describes fully the convex hull of the single-item uncapacitated problem. Some special cases of single-item problems are investigated in Küçükyavuz and Pochet [24] (uncapacitated, backlogging), Pochet and Wolsey [37] (constant capacities), Loparic et al. [26] (uncapacitated, sales and safety stocks), and Constantino [13] (uncapacitated, start-up costs). Atamtürk and Muñoz [4] provide a recent polyhedral study that investigates the bottleneck cover structure in capacitated single-item problems, and Pochet and Wolsey [36] extend some single-item results to the multi-level case. On the other hand, Miller et al. $[32,33]$ provide rare results on multi-item problems with big bucket capacities, where the authors study single-period relaxations and propose valid inequalities. In a recent study, Levi et al. [25] study a version of the capacitated multi-item problem and they propose an approximation algorithm based on generating flow cover inequalities and randomized rounding.

A compact extended reformulation for production planning is the facility location reformulation of Krarup and Bilde [23], which defines the convex hull of the uncapacitated single-item problem when projected to original variable space. Eppen and Martin [15] study the shortest path reformulation, which is of smaller size compared to facility location reformulation. Rardin and Wolsey [39] investigate the multi-commodity reformulation for fixed-charge network problems. Belvaux and Wolsey [8] and Wolsey [50] are recent studies about reformulations and modeling issues. Anily et al. [3] provide tight reformulations for some special cases of the multi-item problem with joint setups.

Finally, we note that mathematical programming results on production planning problems are not only limited to these two approaches. Lagrangian relaxation has been used by Billington et al. [9] in a Branch\&Bound scheme, as well as in the heuristic approach of Thizy and Van Wassenhove [45]. On the other hand, Dantzig-Wolfe decomposition has been in use since the paper of Manne [28], with advancements of Bitran and Matsuo [10] and very recently of Degraeve and Jans [14]. We refer the interested reader to Buschkühl et al. [12] for a thorough and very recent review.

\subsection{Motivation and Organization of the Paper}

In spite of this research, big bucket production planning problems remain hard to solve. Part of the reason for this is that most previous research focuses on developing and using results for single-item models, which are not sufficient to capture the fundamental sources of complexity of big bucket problems. The 
primary goals of this paper are to evaluate the strength of the relaxations defined by different mathematical programming techniques and to investigate why big bucket production planning problems are hard to solve in practice. More specifically, we are not primarily interested in extending single-item results to general production planning problems, but we want to discover relationships between different methods for generating lower bounds and the fundamental substructures that often make these methods insufficient to solve these problems well. We will consider all known methods for generating lower bounds of which we are aware, and we will investigate previously untried methods as well.

In Section 2, we provide a comprehensive survey of lower bounding methods presented in previous research, and we discuss previously untested methods as well. Section 3 is devoted to theoretical comparisons of different techniques, which can provide structural insight into multi-level big bucket problems. In Section 4 , we present extensive computational comparisons obtained using widely used data sets. We conclude with future directions in Section 5.

\section{Valid Inequalities, Reformulations, and Relaxations}

In this section we discuss different approaches to obtain lower bounds. These methods vary from defining valid inequalities and reformulations to the use of Lagrangian relaxation.

\subsection{Valid Inequalities}

The first technique we consider is the use of $(\ell, S)$ inequalities of Barany et al. [5] defined for single-item problems, and generalized by Pochet and Wolsey [36] to multi-level problems using the echelon reformulation. These can be defined as follows:

$$
\sum_{t \in S} x_{t}^{i} \leq \sum_{t \in S} D_{t, \ell}^{i} y_{t}^{i}+E_{\ell}^{i} \quad \ell \in[1, N T], i \in[1, N I], S \subseteq[1, \ell]
$$

Since these inequalities are valid for the single-item submodels defined by each item, they are valid for the multi-item problem as well. Although there is an exponential number of these inequalities, a simple polynomial separation algorithm exists as shown in Barany et al. [6], see Algorithm 1. As will be discussed later, there exist stronger formulations for the multi-level problem than that provided by using the $(\ell, S)$ inequalities alone, but $(\ell, S)$ inequalities have good practical use, especially when considering large problems.

The feasible region associated with this formulation can be defined as $X_{L S}=\{(x, y, E) \mid(4)-(7),(11)-(13),(15)\}$, and the problem can be defined as $Z_{L S}=\min \left\{(14) \mid(x, y, E) \in X_{L S}\right\}$. 
Algorithm 1: $(\ell, S)$ separation

Input: LP relaxation solution $\left(x^{*}, y^{*}, E^{*}\right)$

Output: Violated $(\ell, S)$ inequalities

for $\mathrm{i}=1$ to $\mathrm{NI}$

for $\ell=1$ to NT

Initialize $S \leftarrow\{\}$

for $\mathrm{t}=1$ to $\ell$

if $x_{t}^{* i}>D_{t, \ell}^{i} y_{t}^{* i}$

$S \leftarrow S \cup\{t\}$

if $\sum_{t \in S} x_{t}^{* i}>\sum_{t \in S} D_{t, \ell}^{i} y_{t}^{* i}+E_{\ell}^{* i}$

Add the violated $(\ell, S)$ inequality

\subsection{Reformulations}

The next technique we consider is the facility location reformulation, originally defined by Krarup and Bilde [23] for the single-item problem. This reformulation divides production according to which period it is intended for. This requires first defining new variables $u_{t, t^{\prime}}^{i}$, which indicate the production of item $i$ in period $t$ to satisfy the demand of period $t^{\prime}$, where $t^{\prime} \geq t$. The following constraints should be added into the basic formulation to finalize the reformulation:

$$
\begin{array}{ll}
u_{t, t^{\prime}}^{i} \leq D_{t^{\prime}}^{i} y_{t}^{i} & t \in[1, N T], t^{\prime} \in[t, N T], i \in[1, N I] \\
\sum_{t=1}^{t^{\prime}} u_{t, t^{\prime}}^{i}=D_{t^{\prime}}^{i} & t^{\prime} \in[1, N T], i \in[1, N I] \\
x_{t^{\prime}}^{i}=\sum_{t=t^{\prime}}^{N T} u_{t^{\prime}, t}^{i} & t^{\prime} \in[1, N T], i \in[1, N I] \\
u \geq 0 &
\end{array}
$$

This formulation adds $O\left(N T^{2} N I\right)$ variables and $O\left(N T^{2} N I\right)$ constraints to the problem. One advantage of using the new variables $u_{t, t^{\prime}}^{i}$ is that we can rewrite the capacity constraint (4) as follows:

$$
\sum_{i=1}^{N I}\left(a_{k}^{i}\left(\sum_{t^{\prime}=t}^{N T} u_{t, t^{\prime}}^{i}\right)+S T_{k}^{i} y_{t}^{i}\right) \leq C_{t}^{k} \quad t \in[1, N T], k \in[1, N K]
$$

This, along with constraints (16), can considerably help a state-of-the-art MIP solver generate knapsack cover cuts. Specifically, note that by adding $\sum_{i=1}^{N I} a_{k}^{i} D_{t, N T}^{i} y_{t}^{i}$ on both sides and after rearranging the terms, (20) can be rewritten as

$$
\sum_{i=1}^{N I}\left(a_{k}^{i} D_{t, N T}^{i}+S T_{k}^{i}\right) y_{t}^{i} \leq C_{t}^{k}+\left(\sum_{i=1}^{N I} \sum_{t^{\prime}=t}^{N T} a_{k}^{i}\left(D_{t^{\prime}}^{i} y_{t}^{i}-u_{t, t^{\prime}}^{i}\right)\right)
$$


For each fixed pair of $(t, k)$, and for any subsets $\mathcal{I} \subseteq\{1, \ldots, N I\}$ and $\mathcal{T} \subseteq$ $\{t, \ldots, N T\}$, we may generate cover cuts for each of the following continuous 0-1 knapsack constraints (which is obtained in the same fashion as (21), but only for the subsets $\mathcal{I}$ and $\mathcal{T}$ and the "continuous variable" is highlighted in parenthesis on the right hand side):

$$
\sum_{i \in \mathcal{I}}\left(a_{k}^{i}\left(\sum_{t^{\prime} \in \mathcal{T}} D_{t^{\prime}}^{i}\right)+S T_{k}^{i}\right) y_{t}^{i} \leq C_{t}^{k}+\left(\sum_{i \in \mathcal{I}} \sum_{t^{\prime} \in \mathcal{T}} a_{k}^{i}\left(D_{t^{\prime}}^{i} y_{t}^{i}-u_{t, t^{\prime}}^{i}\right)\right)
$$

Note that because of (16), the expression in the parenthesis on the righthand side of (21) or (22) can be considered as a single nonnegative continuous variable. Binary knapsack constraints with a single nonnegative continuous variable were studied by Marchand and Wolsey [29,30] (see also Richard et al. $[40,41])$. Commercial solvers use the kinds of results they present to efficiently find subsets $\mathcal{I}$ and $\mathcal{T}$ and generate cover cuts that will approximate $\operatorname{conv}\left(X_{K N}^{(t, k)}\right)$, where $X_{K N}^{(t, k)}=\{(y, u) \mid(6),(16),(19),(20)\}$ is the feasible region of the intersection of these continuous 0-1 knapsack problems for a fixed $(t, k)$ pair. Note that we can also define it as $X_{K N}^{(t, k)}=\operatorname{proj}_{y, u} \bar{X}_{K N}^{(t, k)}$ with $\bar{X}_{K N}^{(t, k)}=\{(x, y, E, u) \mid(6),(16),(19),(20),(18),(11)\}$, just for the convenience of having it in higher dimension. Related to $\bar{X}_{K N}^{(t, k)}$, we will define $\bar{X}_{K N}^{(t, k,\{t(i)\})}$, for which we first choose a $t(i) \in[t, N T]$ for all $i \in[1, N I]$, for a given $t$. Then, we define

$$
\begin{array}{ll}
u_{t, t_{1}}^{i} \leq D_{t_{1}}^{i} y_{t}^{i} & t_{1} \in[t, N T], i \in[1, N I] \\
u_{t_{1}, t_{2}}^{i} \leq D_{t_{2}}^{i} y_{t_{1}}^{i} & t_{1} \in[t+1, t(i)], t_{2} \in\left[t_{1}, t(i)\right], \\
x_{t}^{i}=\sum_{t_{1}=t}^{N T} u_{t, t_{1}}^{i} & i \in[1, N I] \\
E_{t-1}^{i}=\sum_{t_{1}=1}^{t-1} \sum_{t_{2}=t}^{N T} u_{t_{1}, t_{2}}^{i} & i \in[1, N I] \\
x_{t}^{i}+E_{t-1}^{i}+\sum_{t_{1}=t+1}^{t(i)} \sum_{t_{2}=t_{1}}^{t(i)} u_{t_{1}, t_{2}}^{i} \geq D_{t, t(i)}^{i} & i \in[1, N I]
\end{array}
$$

Then, $\bar{X}_{K N}^{(t, k,\{t(i)\})}=\{(x, y, E, u) \mid(6),(19),(20),(23)-(27)\}$. Note that we will use this explicit definition for the purposes of proving a key proposition in the next section.

On a separate note, basic continuous cover inequalities can also be generated as MIR inequalities, which are known to be effective for general mixed integer programs (see e.g. Günlük and Pochet [20]). Of course, our approach will increase the problem size and it might easily become so large that it cannot be solved to optimality in an acceptable time. However, using this approach for the purpose of generating lower bounds can yield insights into the structure of 
our problems. This idea was initially suggested for single-level, single-machine problems by Van Vyve ${ }^{1}$. To the best of our knowledge, this approach has not been tested for multi-level problems before.

The feasible region associated with the facility location reformulation can be defined as $X_{F L}=\{(x, y, E, u) \mid(5)-(7),(11)-(13),(16)-(20)\}$, and the associated problem as $Z_{F L}=\min \left\{(14) \mid(x, y, E, u) \in X_{F L}\right\}$. On the other hand, generating all cover cuts approximates $\bigcap_{t=1}^{N T} \bigcap_{k=1}^{N K} \operatorname{conv}\left(X_{K N}^{(t, k)}\right)$, which is an approximation for $\operatorname{conv}\left(\bigcap_{t=1}^{N T} \bigcap_{k=1}^{N K} X_{K N}^{(t, k)}\right)$. This leads us to define the polyhedron:

$$
X_{F L}^{K N}=\{(x, y, E, u) \mid(5),(7),(11)-(13),(17),(18)\} \cap \operatorname{conv}\left(\bigcap_{t=1}^{N T} \bigcap_{k=1}^{N K} X_{K N}^{(t, k)}\right)
$$

and the associated problem $Z_{F L}^{K N}=\min \left\{(14) \mid(x, y, E, u) \in X_{F L}^{K N}\right\}$.

Next, we discuss the single-period relaxation of Miller et al. [32,33], called as $P I$ (Preceding Inventory). To describe the single-period formulation, for a given machine $k \in[1, N K]$ and a given time period $t \in[1, N T]$, we choose a time period $t(i) \geq t$ for each $i \in[1, N I]$. Then we define

$$
\begin{array}{rlrl}
S^{i}=E_{t-1}^{i}+\sum_{\hat{t}=t+1}^{t(i)} D_{\hat{t}, t(i)}^{i} y_{\hat{t}}^{i} & i \in[1, N I] \\
D^{i}=D_{t, t(i)}^{i} & i \in[1, N I]
\end{array}
$$

Then, the single-period formulation can be written as follows:

$$
\begin{array}{ll}
x_{t}^{i}+S^{i} \geq D^{i} & i \in[1, N I] \\
x_{t}^{i} \leq M_{t}^{i} y_{t}^{i} & i \in[1, N I] \\
\sum_{i=1}^{N I}\left(a_{k}^{i} x_{t}^{i}+S T_{k}^{i} y_{t}^{i}\right) \leq C_{t}^{k} & \\
x_{t}^{i}, S^{i} \geq 0 & i \in[1, N I] \\
y_{t}^{i} \in\{0,1\} & i \in[1, N I]
\end{array}
$$

We can define $X_{P I}^{(t, k,\{t(i)\})}=\{(x, y, S) \mid(28)-(32)\}$ as the feasible region associated with a set of $t(i)$ values, and $X_{P I}^{(t, k)}=\bigcap_{\{t(i)\}} X_{P I}^{(t, k,\{t(i)\})}$ represents the feasible region for a given $(t, k)$ pair. Note the similarity between this feasible region and $X_{K N}^{(t, k)}$ we discussed earlier. Miller et al. [32,33] define valid inequalities (namely cover and reverse cover inequalities) for $P I$, which are naturally valid for the original problem as well, and these inequalities can be seen as an approximation for $\operatorname{conv}\left(X_{P I}^{(t, k)}\right)$, which is of interest in our context as providing a lower bound for the original problem when all single-period relaxations are considered for a problem.

1 Personal communication. 
Next, we define the shortest path reformulation of Eppen and Martin [15]. In this formulation, which was originally defined for single-item uncapacitated models, the variables $z_{t, t^{\prime}}^{i}$ are 1 if production of $i$ in period $t$ satisfies all the demand for $i$ in periods $t, \ldots, t^{\prime}$ but not beyond $t^{\prime}$, and 0 otherwise. Note, as a result of the constraints (5) and zero initial inventories, the relationship between the new and original variables is as follows:

$$
x_{t}^{i}=\sum_{t^{\prime}=t}^{N T} D_{t, t^{\prime}}^{i} z_{t, t^{\prime}}^{i} \quad t \in[1, N T], i \in[1, N I]
$$

For the multi-level capacitated problem, we do not have the same optimality properties that we have for the single-item problem; we therefore let the $z$ variables take fractional values as they represent "the fraction of demand in periods $t, \ldots, t^{\prime}$ satisfied by production in period $t "$ ". Also, using the echelon inventory holding costs $H_{t}^{i}$, we define total inventory costs $c_{t, t^{\prime}}^{i}=D_{t, t^{\prime}}^{i} \sum_{j=t}^{N T} H_{j}^{i}$. The formulation is then as follows:

$$
\begin{aligned}
& \min \sum_{t=1}^{N T} \sum_{i=1}^{N I} f_{t}^{i} y_{t}^{i}+\sum_{t=1}^{N T} \sum_{t^{\prime}=t}^{N T} \sum_{i=1}^{N I} c_{t, t^{\prime}}^{i} z_{t, t^{\prime}}^{i} \\
& \text { s.t. } 1=\sum_{t=1}^{N T} z_{1, t}^{i} \\
& i \in[1, N I] \\
& \sum_{t=1}^{t^{\prime}-1} z_{t, t^{\prime}-1}^{i}=\sum_{t=t^{\prime}}^{N T} z_{t^{\prime}, t}^{i} \\
& t^{\prime} \in[2, N T], i \in[1, N I] \\
& \sum_{t^{\prime}=t}^{N T} z_{t, t^{\prime}}^{i} \leq y_{t}^{i} \\
& t \in[1, N T], i \in[1, N I] \\
& \sum_{i=1}^{N I}\left(S T_{k}^{i} y_{t}^{i}+a_{k}^{i} \sum_{t^{\prime}=t}^{N T} D_{t, t^{\prime}}^{i} z_{t, t^{\prime}}^{i}\right) \leq C_{t}^{k} \quad t \in[1, N T], k \in[1, N K] \\
& \sum_{t=1}^{t^{\prime}} \sum_{\hat{t}=t}^{N T}\left(D_{t, \hat{t}}^{i} z_{t, \hat{t}}^{i}-\sum_{j \in \delta(i)} r^{i j} D_{t, \hat{t}}^{j} \hat{z}_{t, \hat{t}}^{j}\right) \geq d_{1, t^{\prime}}^{i} \quad t^{\prime} \in[1, N T], i \in[1, N I] \\
& z \geq 0 \\
& y \in\{0,1\}^{N T x N I}
\end{aligned}
$$

The constraints (35) and (36) are the flow balance constraints, (37) provide the relationship between the linear and binary variables, (38) is the capacity constraint, (39) ensures the relationship between different levels, and finally (40) and (41) provide the nonnegativity and integrality constraints. Note that for our multi-level problem, we derive the constraint (39) as follows: Using (11) and (12), and the assumption of zero initial inventory, we obtain

$$
\sum_{t=1}^{t^{\prime}}\left(x_{t}^{i}-D_{t}^{i}\right) \geq \sum_{t=1}^{t^{\prime}} \sum_{j \in \delta(i)} r^{i j}\left(x_{t}^{j}-D_{t}^{j}\right)
$$


Substituting (33) into (42) and rewriting results in (39). Note that this formulation adds as many variables as the facility location reformulation, but number of constraints is only $O(N T \times N I)$. However, this formulation is not necessarily easier to solve, in part because the new constraints are comparatively dense and the coefficients on the new variables comparatively large.

The feasible region associated with this formulation can be defined as $X_{S P}=\{(y, z) \mid(35)-(41)\}$, and the problem can be defined as $Z_{S P}=\min \{(34) \mid$ $\left.(y, z) \in X_{S P}\right\}$. Part of our motivation for completely substituting the $x$ and $E$ variables out of the formulation is that relaxing the constraints (35), (36), and (39) decomposes the problem into NT distinct subproblems, one for each time period (an analogous observation was first made for single-level problems by Jans and Degraeve [21]). We will discuss this property in more detail later.

Next, we consider the multi-commodity reformulation proposed by Rardin and Wolsey [39]. This approach is originally described for fixed-charge network flow problems. Like the facility location reformulation, it divides production using destination information, but since we have multiple levels, it also includes information about which end-item in the BOM it is produced for. Stock variables are also divided in a similar fashion. Thus, the new variables $w_{t, t^{\prime}}^{i, j}$ indicate production of item $i$ in period $t$ to satisfy the demand of end-item $j$ in period $t^{\prime}, t^{\prime} \geq t$, and the new variables $v_{t, t^{\prime}}^{i, j}$ indicate the inventory of item $i$ held over at the end of period $t$ to satisfy demand of end-item $j$ in period $t^{\prime}$, $t^{\prime}>t$. The following constraints should be added to the basic formulation to finalize the reformulation:

$$
\begin{aligned}
& x_{t^{\prime}}^{i}=\sum_{t=t^{\prime}}^{N T} \sum_{j \in e n d p} w_{t^{\prime}, t}^{i, j} \\
& t^{\prime} \in[1, N T], i \in[1, N I] \\
& w_{t, t^{\prime}}^{i, j} \leq r^{i j} d_{t^{\prime}}^{j} y_{t}^{i} \\
& t \in[1, N T], t^{\prime} \in[t, N T], \\
& i \in[1, N I], j \in \operatorname{endp} \\
& v_{t-1, t}^{i, i}+w_{t, t}^{i, i}=d_{t}^{i} \\
& t \in[1, N T], i \in e n d p \\
& v_{t-1, t^{\prime}}^{i, i}+w_{t, t^{\prime}}^{i, i}=v_{t, t^{\prime}}^{i, i} \\
& t \in[1, N T-1], t^{\prime} \in[t+1, N T] \text {, } \\
& i \in \operatorname{endp} \\
& v_{t-1, t}^{i, q}+w_{t, t}^{i, q}=\sum_{j \in \delta(i)} r^{i j} w_{t, t}^{j, q} \quad t \in[1, N T], i \in[1, N I] \backslash e n d p, \\
& q \in e n d p \\
& v_{t-1, t^{\prime}}^{i, q}+w_{t, t^{\prime}}^{i, q}=v_{t, t^{\prime}}^{i, q}+\sum_{j \in \delta(i)} r^{i j} w_{t, t^{\prime}}^{j, q} \quad t \in[1, N T-1], t^{\prime} \in[t+1, N T], \\
& i \in[1, N I] \backslash e n d p, q \in e n d p
\end{aligned}
$$

The constraints (43) indicate the relation between the new and old variables, (44) provide the relationship between the linear and binary variables, 
(45) and (46) are demand flow balance constraints for end items, (47) and (48) are demand flow balance constraints for non-end items, and finally (49) provide the nonnegativity constraints. This reformulation introduces $O\left(N T^{2} N I^{2}\right)$ additional variables and $O\left(N T^{2} N I^{2}\right)$ additional constraints. This is the main disadvantage of this reformulation, which can easily become computationally intractable as the problem size grows. However, it is the tightest compact, i.e., polynomial size, reformulation that we know for the problems in question.

The feasible region associated with this formulation can be defined as $X_{M C}=\{(x, y, E, w, v) \mid(4)-(7),(11)-(13),(43)-(49)\}$, and the problem can be defined as $Z_{M C}=\min \left\{(14) \mid(x, y, E, w, v) \in X_{M C}\right\}$.

\subsection{Relaxations}

Next, we discuss three approaches that employ Lagrangian relaxation to obtain structured subproblems and from those lower bounds for the original problem. The first approach is to relax the capacity constraints (4), and obtain

$$
\begin{aligned}
L R_{1}(\lambda)= & \min \sum_{t=1}^{N T} \sum_{i=1}^{N I} f_{t}^{i} y_{t}^{i}+\sum_{t=1}^{N T} \sum_{i=1}^{N I} H^{i} E_{t}^{i} \\
& -\sum_{t=1}^{N T} \sum_{k=1}^{N K} \lambda_{t}^{k}\left(C_{t}^{k}-\left(\sum_{i=1}^{N I} a_{k}^{i} x_{t}^{i}+S T_{k}^{i} y_{t}^{i}\right)\right) \\
& \text { subject to }(x, y, E) \in X_{L R 1}
\end{aligned}
$$

where $X_{L R 1}=\{(x, y, E) \mid(5)-(7),(11)-(13)\}$. Thus, the Lagrangian subproblem is a multi-item, multi-level uncapacitated production planning problem. The Lagrangian dual problem is

$$
L D_{1}=\max _{\lambda \geq 0} L R_{1}(\lambda)
$$

The next Lagrangian relaxation approach relaxes the constraints linking separate levels, i.e. constraints (12), to obtain

$$
\begin{aligned}
L R_{2}(\mu)= & \min \sum_{t=1}^{N T} \sum_{i=1}^{N I} f_{t}^{i} y_{t}^{i}+\sum_{t=1}^{N T} \sum_{i=1}^{N I} H^{i} E_{t}^{i} \\
& -\sum_{t=1}^{N T} \sum_{i=1}^{N I} \mu_{t}^{i}\left(E_{t}^{i}-\sum_{j \in \delta(i)} r^{i j} E_{t}^{j}\right) \\
& \text { subject to }(x, y, E) \in X_{L R 2}
\end{aligned}
$$

where $X_{L R 2}=\{(x, y, E) \mid(4)-(7),(11),(13)\}$. The Lagrangian subproblem therefore decomposes into $N K$ disjoint multi-item, big bucket single-machine problems, one for each machine. The Lagrangian dual problem becomes

$$
L D_{2}=\max _{\mu \geq 0} L R_{2}(\mu)
$$


Finally, the last Lagrangian approach extends the work of Jans and Degraeve [21] for single-level problems, which itself uses the shortest path reformulation of Eppen Martin [15]. Jans and Degraeve [21] simply relaxed the constraints linking time periods, yielding disjoint single-period subproblems. However, the problem in the multi-level case is that the constraints linking levels also involve multiple periods. Therefore, decomposing the problem into disjoint subproblems for each period is not possible, unless all constraints linking levels are also dualized. We dualize the constraints (35), (36) and (39) in the shortest path reformulation to obtain

$$
\begin{aligned}
L R_{3}(\beta, \gamma)= & \min \sum_{t=1}^{N T} \sum_{i=1}^{N I} f_{t}^{i} y_{t}^{i}+\sum_{t=1}^{N T} \sum_{t^{\prime}=t}^{N T} \sum_{i=1}^{N I} c_{t, t^{\prime}}^{i} z_{t, t^{\prime}}^{i}-\sum_{i=1}^{N T} \beta_{1}^{i}\left(1-\sum_{t=1}^{N T} z_{1, t}^{i}\right) \\
& -\sum_{i=1}^{N I} \sum_{t^{\prime}=2}^{N T} \beta_{t^{\prime}}^{i}\left(\sum_{t=1}^{t^{\prime}-1} z_{t, t^{\prime}-1}^{i}-\sum_{t=t^{\prime}}^{N T} z_{t^{\prime}, t}^{i}\right) \\
& -\sum_{i=1}^{N I} \sum_{t^{\prime}=1}^{N T} \gamma_{t^{\prime}}^{i}\left(\sum_{t=1}^{t^{\prime}} \sum_{\hat{t}=t}^{N T}\left(D_{t, \hat{t}}^{i} z_{t, \hat{t}}^{i}-\sum_{j \in \delta(i)} r^{i j} D_{t, \hat{t}}^{j} z_{t, \hat{t}}^{j}\right)-d_{1, t^{\prime}}^{i}\right) \\
& \text { subject to }(y, z) \in X_{L R 3}
\end{aligned}
$$

where $X_{L R 3}=\{(y, z) \mid(37),(38),(40),(41)\}$. It is easy to note that the Lagrangian subproblem decomposes into $N K \times N T$ disjoint capacitated multiitem, single-machine, single-period problems. The Lagrangian dual is

$$
L D_{3}=\max _{\gamma \geq 0, \beta} L R_{3}(\beta, \gamma)
$$

In the next section we provide theoretical comparisons for the various approaches we have described.

\section{Exploring Relationships}

Let the superscript $L P$ indicate the LP relaxation of a problem, i.e., the binary variables $y$ relaxed to be continuous with the bounds $0 \leq y \leq 1$. For example, $Z_{L S}^{L P}$ is the problem $Z_{L S}$ with the integrality requirements for $y$ variables relaxed. Similarly, $X_{L S}^{L P}$ is the polyhedron of the LP relaxation of $X_{L S}$.

Theorem 1 (Akartunalı and Miller [1]) $Z_{L S}^{L P}=Z_{F L}^{L P}=Z_{S P}^{L P}$, i.e., the $(\ell, S)$ inequalities, the facility location reformulation, and the shortest path reformulation all provide the same lower bound for the original problem.

For the proof of the theorem, please refer to Akartunall [2]. The proof uses Lagrangian duality and the fact that all these formulations provide equal lower bounds in the single-item case. See Krarup and Bilde [23], Eppen and Martin [15], and Barany et al. [6] for the convex hull and integrality proofs in the single-item case. 
Theorem $2 Z_{M C}^{L P} \geq Z_{F L}^{L P}$, i.e., the multi-commodity reformulation provides a lower bound that is at least as strong as that provided by the facility location reformulation. If the problem consists of a single level, then $Z_{M C}^{L P}=Z_{F L}^{L P}$.

Although this result has been known by at least some researchers since the publication of Rardin and Wolsey [39], it has never been formally stated and proven, to the best of our knowledge. We therefore provide a proof for the sake of completeness.

Proof We will prove this by showing that $\operatorname{proj}_{x, y, E}\left(X_{M C}^{L P}\right) \subseteq \operatorname{proj}_{x, y, E}\left(X_{F L}^{L P}\right)$ for the multi-level case. Let $\left(v^{*}, w^{*}, x^{*}, y^{*}, E^{*}\right) \in X_{M C}^{L P}$. First, observe that we can eliminate $v^{*}$ and rewrite (45)-(48) in terms of $w^{*}$, as follows:

$$
\sum_{t=1}^{t=t^{\prime}} w_{t, t^{\prime}}^{* i, j}=r^{i j} d_{t^{\prime}}^{j} \quad t^{\prime} \in[1, N T], i \in[1, N I], j \in e n d p
$$

Now, let

$$
u_{t, t^{\prime}}^{* i}=\sum_{j \in e n d p} w_{t, t^{\prime}}^{* i j}
$$

Obviously $u^{*} \geq 0$ since $w^{*} \geq 0$. Since $w^{*}$ satisfies (43), $x_{t}^{* i}=\sum_{t^{\prime}=t}^{N T} u_{t, t^{\prime}}^{* i}$. Similarly, summing (56) over $j \in e n d p$, we obtain $\sum_{t=1}^{t^{\prime}} u_{t, t^{\prime}}^{* i}=\sum_{j \in e n d p} r^{i j} d_{t^{\prime}}^{j}$ $=D_{t^{\prime}}^{i}$, where the second equation follows from the definition of echelon demand (9). Finally, using (44) and (57), we obtain $u_{t, t^{\prime}}^{* i}=\sum_{j \in e n d p} w_{t, t^{\prime}}^{* i j} \leq$ $\left(\sum_{j \in e n d p} r^{i j} d_{t^{\prime}}^{j}\right) y_{t}^{* i}=D_{t^{\prime}}^{i} y_{t}^{* i}$. This shows that $\left(u^{*}, x^{*}, y^{*}, E^{*}\right) \in X_{F L}^{L P}$. Hence, $\operatorname{proj}_{x, y, E}\left(X_{M C}^{L P}\right) \subseteq \operatorname{proj}_{x, y, E}\left(X_{F L}^{L P}\right)$.

The second part of the theorem can also be shown using the same technique as in the proof of first theorem, i.e., using Lagrangian duality and the fact that the multi-commodity reformulation and the facility location reformulation provide equivalent lower bounds in the single-item case (see Eppen and Martin [15] and Barany et al. [6]).

This theorem shows us theoretically that the multi-commodity reformulation is stronger than the formulation defined by adding $(\ell, S)$ inequalities, the facility location reformulation, and the shortest path reformulation. In the next section, we will computationally address the question of "how much stronger" for a variety of test problems.

So far we have made comparisons of different polyhedral approaches. Also interesting are the relationships between the Lagrangian approaches and these reformulations, as we investigate in the following results.

Theorem $3 Z_{M C}^{L P} \leq L D_{1}$.

In words, the lower bound obtained by the Lagrangian that relaxes the capacity constraints is at least as strong as the lower bound obtained by multicommodity reformulation. 
Proof By the theorem related to the strength of the Lagrangian dual (see e.g. Theorem 10.3 of Wolsey [49]),

$$
L D_{1}=\min \{(14) \mid(x, y, E) \in(4) \cap \operatorname{conv}((5)-(7),(11)-(13))\}
$$

On the other hand,

$$
\begin{aligned}
Z_{M C}^{L P}= & \min \{(14) \mid(x, y, E, w, v) \in(4) \cap\{((5),(7),(11)-(13),(43)-(49)) \\
& \cap \operatorname{conv}((6))\}\}
\end{aligned}
$$

Observe that

$$
\begin{aligned}
& \{(x, y, E) \in \operatorname{conv}((5)-(7),(11)-(13))\} \subseteq \\
& \operatorname{proj}_{x, y, E}\{(x, y, E, w, v) \in\{((5),(7),(11)-(13),(43)-(49)) \cap \operatorname{conv}((6))\}\}
\end{aligned}
$$

This follows because conv $((5)-(7),(11)-(13))$ has integer extreme points because the polyhedron is the convex hull of an integer feasible region. On the other hand, $\{((5),(7),(11)-(13),(43)-(49)) \cap \operatorname{conv}((6))\}$ does not necessarily have integral extreme points. Therefore, $Z_{M C}^{L P} \leq L D_{1}$.

Theorem $4 Z_{F L}^{L P} \leq Z_{F L}^{K N} \leq L D_{2}$.

In words, the lower bound obtained by the Lagrangian that relaxes the level linking constraints is at least as strong as the lower bound obtained by the facility location reformulation strengthened to approximate the knapsack convex hulls.

Proof The first relationship follows from the fact that $Z_{F L}^{K N}$ is obtained by strengthening $Z_{F L}^{L P}$ with additional constraints. For the second relationship, first observe that (using the same theorem as in the previous proof)

$$
L D_{2}=\min \{(14) \mid(x, y, E) \in(12) \cap \operatorname{conv}((4)-(7),(11),(13))\}
$$

Observe also that

$$
\begin{aligned}
& \operatorname{conv}((4)-(7),(11),(13)) \subseteq \\
& \operatorname{proj}_{x, y, E}\left\{\{(x, y, E, u) \mid(5),(7),(11),(13),(17),(18)\} \cap \operatorname{conv}\left(\bigcap_{t=1}^{N T} \bigcap_{k=1}^{N K} X_{K N}^{(t, k)}\right)\right\}
\end{aligned}
$$

This concludes that $Z_{F L}^{K N}$ is not as strong as $L D_{2}$.

As mentioned before, generating cover cuts from (22) only approximates the knapsack polyhedron and hence $Z_{F L}^{K N}$ is the best possible bound that can be obtained by adding cover cuts to the LP relaxation of the facility location reformulation.

Theorem $5 Z_{F L}^{K N}=L D_{3}$.

We will use the following result for the proof of the theorem. 
Lemma 6 (Pochet and Wolsey [35]) All optimal solutions of the singleitem uncapacitated problem formulated using the facility location reformulation have the following property:

$$
\frac{u_{t, t^{\prime}}}{D_{t^{\prime}}} \geq \frac{u_{t, t^{\prime}+1}}{D_{t^{\prime}+1}} \quad t \in[1, N T], t^{\prime} \geq t
$$

Before starting the proof of Theorem 5, let $S_{1}=\bigcap_{t=1}^{N T} \bigcap_{k=1}^{N K} X_{K N}^{(t, k)}=$ $\{(y, u) \mid(6),(16),(19),(20)\}$ and $S_{2}=\{(y, z) \mid(37),(38),(40),(41)\}$. Also let $T_{1}=\left\{(x, y, E, u) \mid((11)-(13),(18)) \cap \operatorname{conv}\left(S_{1}\right)\right\}$ and $T_{2}=\{(x, y, E, z) \mid((11)-$ (13), (33)) $\left.\cap \operatorname{conv}\left(S_{2}\right)\right\}$. Note that $S_{1}$ and $S_{2}$ are integer feasible regions whereas $T_{1}$ and $T_{2}$ are both polyhedra. Then, the proof of Theorem 5 follows.

Proof We will prove this by showing $\operatorname{proj}_{x, y, E}\left(T_{1}\right)=\operatorname{proj}_{x, y, E}\left(T_{2}\right)$, and by the fact that $L D_{3}=\min \left\{(14) \mid(x, y, E, z) \in T_{2}\right\}$.

First, let $\left(x^{*}, y^{*}, E^{*}, u^{*}\right) \in T_{1}$ and hence $\left(x^{*}, y^{*}, E^{*}\right) \in \operatorname{proj}_{x, y, E}\left(T_{1}\right)$. Therefore, $\exists p^{j}=\left(x^{j}, y^{j}, E^{j}, u^{j}\right) \in S_{1}, j \in[1, J]$, such that $\left(x^{*}, y^{*}, E^{*}, u^{*}\right)=$ $\sum_{j=1}^{J} \lambda_{j} p^{j}$ for some $\lambda \geq 0, \sum_{j=1}^{J} \lambda_{j}=1$.

For all $j \in[1, J]$, let $\left\{z_{t N T}^{i}\right\}^{j}=\frac{\left\{u_{t N T}^{i}\right\}^{j}}{D_{N T}^{i}}$, where $t \in[1, N T]$ and $i \in[1, N I]$. Then, define recursively $\left\{z_{t, t^{\prime}}^{i}\right\}^{j}=\frac{\left\{u_{t, t^{\prime}}^{i}\right\}^{j}}{D_{N T}^{i}}-\sum_{\bar{t}=t^{\prime}+1}^{N T}\left\{z_{t, \bar{t}}^{i}\right\}^{j}$, for all $t \in[1, N T]$, $t^{\prime}=N T-1, \ldots, t$ and $i \in[1, N I]$. Since $\sum_{t^{\prime}=t}^{N T} D_{t, t^{\prime}}^{i}\left\{z_{t, t^{\prime}}^{i}\right\}^{j}=\sum_{t^{\prime}=t}^{N T}\left\{u_{t, t^{\prime}}^{i}\right\}^{j}$ and $u^{j}$ satisfies (20), $z^{j}$ satisfies (38). Next, note that

$$
\sum_{t^{\prime}=t}^{N T}\left\{z_{t, t^{\prime}}^{i}\right\}^{j}=\frac{\left\{u_{t, t}^{i}\right\}^{j}}{D_{t}^{i}} \leq\left\{y_{t}^{i}\right\}^{j}
$$

where the last inequality is essentially (16). Finally, using Lemma 6, observe that

$$
\left\{z_{t, t^{\prime}}^{i}\right\}^{j}=\frac{\left\{u_{t, t^{\prime}}^{i}\right\}^{j}}{D_{t^{\prime}}^{i}}-\frac{\left\{u_{t, t^{\prime}+1}^{* i}\right\}^{j}}{D_{t^{\prime}+1}^{i}} \geq 0
$$

Therefore, $\hat{p}^{j}=\left(x^{j}, y^{j}, E^{j}, z^{j}\right) \in S_{2}$, and using the same $\lambda$ as before, $\left(x^{*}, y^{*}, E^{*}, z^{*}\right)=\sum_{j=1}^{J} \lambda_{j} \hat{p}^{j} \in T_{2}$. Hence, $\left(x^{*}, y^{*}, E^{*}\right) \in \operatorname{proj}_{x, y, E}\left(T_{2}\right)$. We conclude therefore that $\operatorname{proj}_{x, y, E}\left(T_{1}\right) \subseteq \operatorname{proj}_{x, y, E}\left(T_{2}\right)$.

Now, let $\left(x^{*}, y^{*}, E^{*}, z^{*}\right) \in T_{2}$ and hence $\left(x^{*}, y^{*}, E^{*}\right) \in \operatorname{proj}_{x, y, E}\left(T_{2}\right)$. Therefore, $\exists q^{k}=\left(x^{k}, y^{k}, E^{k}, z^{k}\right) \in S_{2}, k \in[1, K]$, such that $\left(x^{*}, y^{*}, E^{*}, z^{*}\right)=$ $\sum_{k=1}^{K} \mu_{k} q^{k}$ for some $\mu \geq 0, \sum_{k=1}^{K} \mu_{k}=1$.

For all $k \in[1, K]$, let $\left\{u_{t, t^{\prime}}^{i}\right\}^{k}=D_{t^{\prime}}^{i} \sum_{\bar{t}=t^{\prime}}^{N T}\left\{z_{t, \bar{t}}^{i}\right\}^{k}$, where $t \in[1, N T], t^{\prime} \in$ $[t, N T]$, and $i \in[1, N I]$. Obviously, $u^{k}$ satisfies (19) since $z^{k}$ satisfies (40). Since $\sum_{t^{\prime}=t}^{N T}\left\{u_{t, t^{\prime}}^{i}\right\}^{k}=\sum_{t^{\prime}=t}^{N T} D_{t, t^{\prime}}^{i}\left\{z_{t, t^{\prime}}^{i}\right\}^{k}$ and $z^{k}$ satisfies (38), $u^{k}$ satisfies (20). Finally, note that

$$
\left\{u_{t, t^{\prime}}^{i}\right\}^{k}=D_{t^{\prime}}^{i} \sum_{\bar{t}=t^{\prime}}^{N T}\left\{z_{t, \bar{t}}^{i}\right\}^{k} \leq D_{t^{\prime}}^{i} \sum_{\bar{t}=t}^{N T}\left\{z_{t, \bar{t}}^{i}\right\}^{k} \leq D_{t^{\prime}}^{i}\left\{y_{t}^{i}\right\}^{k}
$$


where the last inequality follows from (37).

Therefore, $\hat{q}^{k}=\left(x^{k}, y^{k}, E^{k}, u^{k}\right) \in S_{1}$, and using the same $\mu$ as before, $\left(x^{*}, y^{*}, E^{*}, u^{*}\right)=\sum_{k=1}^{K} \mu_{k} \hat{q}^{k} \in T_{1}$. Hence, $\left(x^{*}, y^{*}, E^{*}\right) \in \operatorname{proj}_{x, y, E}\left(T_{1}\right)$. Therefore, $\operatorname{proj}_{x, y, E}\left(T_{2}\right) \subseteq \operatorname{proj}_{x, y, E}\left(T_{1}\right)$. This concludes the proof.

Corollary $7 L D_{3} \leq L D_{2}$.

The proof for this corollary follows immediately from the Theorems 4 and 5 . This result is our main motivation for skipping $L D_{3}$ in the computational tests discussed in the next section.

Proposition 8 For any given $(t, k)$ pair and set of $\{t(i)\}$ values,

$$
\operatorname{proj}_{x, y, E}\left(\operatorname{conv}\left(X_{P I}^{(t, k,\{t(i)\})}\right)\right)=\operatorname{proj}_{x, y, E}\left(\operatorname{conv}\left(\bar{X}_{K N}^{(t, k,\{t(i)\})}\right)\right)
$$

This result, combined with Corollary 7, is our main motivation for omitting computationally testing the cover and reverse cover inequalities from Miller et al. $[32,33]$ in the next section.

Proof First show $\operatorname{proj}_{x, y, E}\left(\operatorname{conv}\left(\bar{X}_{K N}^{(t, k,\{t(i)\})}\right)\right) \subseteq \operatorname{proj}_{x, y, E}\left(\operatorname{conv}\left(X_{P I}^{(t, k,\{t(i)\})}\right)\right)$ for a given $(t, k)$ pair and set of $\{t(i)\}$ values. Let $\left(x^{*}, y^{*}, E^{*}, u^{*}\right) \in$ $\operatorname{conv}\left(\bar{X}_{K N}^{(t, k,\{t(i)\})}\right)$. Then, we define $S^{* i}=E_{t-1}^{* i}+\sum_{\hat{t}=t+1}^{t(i)} D_{\hat{t}, t(i)}^{i} y_{\hat{t}}^{* i}$. It is easy to observe that $\left(x^{*}, y^{*}, S^{*}\right) \in \operatorname{conv}\left(X_{P I}^{(t, k,\{t(i)\})}\right)$.

Next we prove $\operatorname{proj}_{x, y, E}\left(\operatorname{conv}\left(X_{P I}^{(t, k,\{t(i)\})}\right)\right) \subseteq \operatorname{proj}_{x, y, E}\left(\operatorname{conv}\left(\bar{X}_{K N}^{(t, k,\{t(i)\})}\right)\right)$ for any given $(t, k)$ pair and set of $\{t(i)\}$ values. First, let $\left(x^{*}, y^{*}, S^{*}\right) \in$ $\operatorname{conv}\left(X_{P I}^{(t, k,\{t(i)\})}\right)$. We define first $u_{t_{1}, t_{2}}^{* i}=D_{t_{2}}^{i} y^{* i}{ }_{t_{1}}$ for all $t_{1} \in[t+1, t(i)]$ and $t_{2} \in\left[t_{1}, t(i)\right]$. Then, we define $E_{t-1}^{* i}=\left(S^{* i}-\sum_{\hat{t}=t+1}^{t(i)} D_{\hat{t}, t(i)}^{i} y_{\hat{t}}^{* i}\right)^{+}$. Finally, define $u_{t, t^{\prime}}^{* i}=\left(\min \left\{D_{t^{\prime}}^{i} y_{t}^{* i}, x_{t}^{* i}-\sum_{\bar{t}=t}^{t^{\prime}-1} u_{t, \bar{t}}^{* i}\right\}\right)^{+}$for all $t^{\prime} \in[t, t(i)]$, where they are calculated in the increasing order of $t^{\prime}$. Then, we can observe that $\left(x^{*}, y^{*}, E^{*}, u^{*}\right) \in \operatorname{conv}\left(\bar{X}_{K N}^{(t, k, t(i))}\right)$.

\section{Computational Results}

\subsection{Overview}

In order to provide diversified results, we used the following test instances for our computations:

- TDS instances: These test problems originate from Tempelmeier and Derstroff [44] and Stadtler [43]. These include overtime variables in addition to the formulation in Section 2. Sets $\mathrm{A}+$ and $\mathrm{B}+$ involve problems with 10 items and 24 periods, and sets $\mathrm{C}$ and $\mathrm{D}$ involve problems with 40 items and 16 periods. Sets B+ and D include setup times. We chose the hardest instances from each data set for our computations, i.e., for each data set, we picked 10 assembly and 10 general instances with the highest duality gaps according to results of Stadtler [43]. 
- LOTSIZELIB instances: These are the multi-level instances of LOTSIZELIB [27]. These include big bucket capacities, and backlogging is also allowed. The problems vary between $40 \mathrm{item}$, single end-item problems and 15 item, 3 end-item problems. All problems have 12 periods.

- Multi-LSB instances: We have generated 4 sets of test problems based on the problem family described in Simpson and Erenguc [42], each set having 30 instances with low, medium and high variability of demand. We will refer to these sets as SET1, SET2, SET3, and SET4 in the remainder of the paper. The main difference of these instances is that they consider component commonality and hence joint setup variables for each "family of item" (a set of items that are grouped together due to similarity) exist, i.e., setup time of a family of items is exhausted only once as soon as any item from that family is produced. The original BOM structures and holding costs of [42] are preserved, while the setup costs are removed. Moreover, these instances have backlogging variables and hence increase the variety of our test bed. Except for the problems in SET2, which consider a horizon of 24 periods, all the instances have 16 periods. The main difference between SET1, SET2 and SET4 is about resource utilization factors, which are all set over $100 \%$ for obtaining hard problem instances. All problems have 78 items and an assembly BOM structure, and all instances allow backlogging to the last period. For more details about these instances, including a full formulation, see Multi-LSB homepage [34].

Note that average duality gaps after default times (see next section for more detail on "default times") for the test sets of TDS and Multi-LSB are provided in the Table 1 for an overview of problem complexity, where the basic formulation is strengthened with all violated $(\ell, S)$ inequalities generated at the root node of the Branch\&Bound tree using Algorithm 1.

Table 1 Average duality gaps for TDS and Multi-LSB instances

\begin{tabular}{cccccccc}
\hline $\mathrm{A}+$ & $\mathrm{B}+$ & $\mathrm{C}$ & $\mathrm{D}$ & SET1 & SET2 & SET3 & SET4 \\
\hline $25.28 \%$ & $34.21 \%$ & $35.40 \%$ & $364.57 \%$ & $17.40 \%$ & $13.84 \%$ & $236.36 \%$ & $78.87 \%$ \\
\hline
\end{tabular}

The main goal of this section is to computationally test the results we have theoretically proven and to observe how these strength relationships work in practice. This not only provides us with information about how strong the lower bounds actually are but also helps us to understand what prevents us from improving them. All the test instances are run on a PC with an Intel Pentium $42.53 \mathrm{GHz}$ processor and $1 \mathrm{~GB}$ of RAM. All the formulations are implemented using Xpress Mosel (Xpress-MP 2004C, Mosel version 1.4.1).

In evaluating Lagrangians, we do not exactly solve any of the Lagrangian dual problems and solve an approximation instead, as detailed in the next paragraph. The main reason to avoid calculating Lagrangian duals is the significant computational effort needed, as this exact calculation will require subgradi- 
ent optimization to choose the optimal Lagrangian multipliers. Subgradient optimization does not have a guarantee for convergence (e.g. noted by [21]) and it might require a very high number of iterations (each iteration being a Lagrangian relaxation problem) to converge to a bound. This is prohibitive in our case, as Lagrangian problems prove not to be easy to solve to optimality in short computational times: As we will also see later in computational results, even for the smaller TDS test sets of $\mathrm{A}+$ and $\mathrm{B}+$, only one instance (namely AK501432) solved to optimality for the 1st Lagrangian relaxation problem in the given 180 seconds time limit. Moreover, ad-hoc testing of subgradient optimization for Lagrangian duals on a few small $\mathrm{A}+$ an $\mathrm{B}+$ instances did not seem to converge efficiently to a bound within CPU times of 2 to 7 hours.

For the approximation to Lagrangian duals, we first consider a strengthened LP formulation, i.e., the echelon formulation with all violated $(\ell, S)$ inequalities generated at the root node, and then fix the Lagrangian multipliers to the values of the optimal dual variables of the constraints to be relaxed in this formulation. We thus evaluate $L R_{1}\left(\lambda^{*}\right)$ and $L R_{2}\left(\mu^{*}\right)$, respectively, for the optimal dual variables $\lambda^{*}$ of the capacity constraints and the optimal dual variables $\mu^{*}$ of the level-linking constraints, respectively, in order to approximate $L D_{1}$ and $L D_{2}$, respectively. These subproblems themselves are MIPs that, in general, are difficult to solve to optimality, as can be seen in computational results. Nevertheless, any lower bound on the optimal solution of the Lagrangian subproblem MIP is also a lower bound on the Lagrangian dual (and hence the original problem), i.e., $L R_{1}\left(\lambda^{*}\right) \leq L D_{1} \leq Z$ and $L R_{2}\left(\mu^{*}\right) \leq L D_{2} \leq Z$. Moreover, in every instance, for both $L R_{1}\left(\lambda^{*}\right)$ and $L R_{2}\left(\mu^{*}\right)$, the lower bound obtained computationally for the Lagrangian subproblem MIP is at least as strong as the lower bound provided by the original echelon formulation strengthened with $(\ell, S)$ inequalities. We note that this is the only theoretical strength we are aware of for using these multipliers. Finally, although this is a limited computational experience and cannot necessarily generalize to other instances, our ad-hoc testing of subgradient optimization indicated that the bounds obtained using $\lambda^{*}$ and $\mu^{*}$ can be very competitive.

Similarly, as we discussed before, generating cover cuts on top of the facility location reformulation provides only an approximation of $Z_{F L}^{K N}$. Hence, the computational comparisons we provide for these relationships are all based on approximations. However, this still gives us the chance to compare empirical results in addition to theoretically proven relationships.

\subsection{Results}

The detailed results for TDS instances can be found in the "Online Supplement". Note that we obtain the root node solution of the Branch\&Bound tree for $(\ell, S)$ inequalities, all generated through Algorithm 1, and for the multicommodity reformulation (MC), without the effect of any solver cuts. For the facility location reformulation (FL), all the cover cuts generated by the solver 
are added at the root node and this strengthened formulation is used as FL lower bound. For comparison purposes, we also use the lower bound obtained by the heuristic in our companion paper (Akartunalı and Miller [1]), where the lower bound is based on the first iteration of a relax-and-fix framework, i.e., a partial LP relaxation of the original problem. For the Lagrangian relaxations that relax the capacity and level-linking constraints, we use the dual optimal values of the constraints from the strong LP relaxation as multipliers, and we set default times of 180 seconds for $\mathrm{A}+$ and $\mathrm{B}+$ instances, and 500 seconds for $\mathrm{C}$ and $\mathrm{D}$ instances. Note that if a Lagrangian relaxation subproblem $\left(L R_{1}\left(\lambda^{*}\right)\right.$ or $L R_{2}\left(\mu^{*}\right)$, referred as LR1 and LR2 in the discussion, resp.) is not solved to optimality in this preassigned time, the lower and upper bounds (denoted by the functions $L B($.$) and U B($.$) , resp.) of this Lagrangian subproblem pro-$ vide us the range where the actual lower bound of this Lagrangian relaxation lies, since $L B\left(L R_{1}\left(\lambda^{*}\right)\right) \leq L R_{1}\left(\lambda^{*}\right) \leq U B\left(L R_{1}\left(\lambda^{*}\right)\right)$ and $L B\left(L R_{2}\left(\mu^{*}\right)\right) \leq$ $L R_{2}\left(\mu^{*}\right) \leq U B\left(L R_{2}\left(\mu^{*}\right)\right)$ obviously hold, while $L R_{1}\left(\lambda^{*}\right) \leq L D_{1} \leq Z$ and $L R_{2}\left(\mu^{*}\right) \leq L D_{2} \leq Z$. Therefore, we use the lower and upper bounds of Lagrangian subproblems in our discussions. One important remark here is that these upper bounds $U B\left(L R_{1}\left(\lambda^{*}\right)\right)$ and $U B\left(L R_{2}\left(\mu^{*}\right)\right)$ do not provide any information on the original problem $Z$. Finally, note that due to Theorem 1 we omit the shortest path reformulation in our tests.

We review the results in pairwise comparisons, which are summarized in Table 2 (for detailed results, refer to Tables 1-4 of "Online Supplement"). One interesting computational comparison is the relationship we have proven in Theorem 2. As we can see from the detailed results, MC improves the $(\ell, S)$ bound slightly, in general less than $\% 1$. The average improvements from the $(\ell, S)$ inequalities bound to the MC bound, calculated as (MC bound $\ell, S$ bound $) /(\ell, S$ bound) for each test instance, are provided in the column "MC vs. $\ell, S$ ", and these values are around $0.20 \%$. Considering the enormous size of the MC reformulation, these improvements are simply not worth the computational effort. The Lagrangian relaxation LR1 that relaxes the capacity constraints (i.e., $L R_{1}\left(\lambda^{*}\right)$ ) provides in general another slight improvement over the lower bounds of the MC reformulation, as can be seen in the second column of the same table (Column LB under "LR1 vs. MC"), which is calculated in a similar fashion, i.e., $\left(L B\left(L R_{1}\left(\lambda^{*}\right)\right)\right.$ - MC bound)/(MC bound). Note that we also provide averages calculated in the same way using the LR1's upper bounds (Column UB under "LR1 vs. MC"), i.e. $\left(U B\left(L R_{1}\left(\lambda^{*}\right)\right)\right.$ - MC bound)/(MC bound). An interesting observation regarding the problems in set $\mathrm{D}$, where all $L R_{1}\left(\lambda^{*}\right)$ problems are solved to optimality, is that although $L R_{1}\left(\lambda^{*}\right)$ provided improvements over the MC bounds for instances outwith set $\mathrm{D}$, the same effect was not observed in set $\mathrm{D}$ instances. This is due to the fact that $L R_{1}\left(\lambda^{*}\right)$ is only an approximation of $L D_{1}$, and therefore it does not necessarily provide a theoretically stronger bound than $\mathrm{MC}$ bound. However, as these results indicate, $L R_{1}\left(\lambda^{*}\right)$ and $\mathrm{MC}$ bounds are in general very close to each other in our computational results.

On the other hand, as the "FL vs. $\ell, S$ " column of Table 2 indicates, the facility location reformulation with cover cuts added (FL) improves in gen- 
Table 2 Pairwise comparisons of lower bounds and LR gaps for TDS instances

\begin{tabular}{|c|c|c|c|c|c|c|c|c|}
\hline \multirow{2}{*}{$\begin{array}{l}\text { Test } \\
\text { Set }\end{array}$} & \multirow{2}{*}{$\begin{array}{c}\text { MC vs. } \\
\ell, S\end{array}$} & \multicolumn{2}{|c|}{ LR1 vs. MC } & \multirow{2}{*}{$\begin{array}{c}\text { FL vs. } \\
\ell, S\end{array}$} & \multicolumn{2}{|c|}{ LR2 vs. FL } & \multicolumn{2}{|c|}{ LR Gaps } \\
\hline & & $\overline{\mathrm{LB}}$ & UB & & $\overline{\mathrm{LB}}$ & $\mathrm{UB}$ & LR1 & LR2 \\
\hline $\mathrm{A}+$ & $0.29 \%$ & $0.80 \%$ & $2.99 \%$ & $1.81 \%$ & $-0.05 \%$ & $7.44 \%$ & $2.09 \%$ & $6.87 \%$ \\
\hline $\mathrm{B}+$ & $0.28 \%$ & $0.59 \%$ & $3.06 \%$ & $1.37 \%$ & $-0.35 \%$ & $6.23 \%$ & $2.38 \%$ & $6.18 \%$ \\
\hline $\mathrm{C}$ & $0.14 \%$ & $0.20 \%$ & $1.67 \%$ & $0.86 \%$ & $-0.32 \%$ & $6.25 \%$ & $1.44 \%$ & $6.14 \%$ \\
\hline $\mathrm{D}$ & $0.21 \%$ & $-0.06 \%$ & $-0.06 \%$ & $0.45 \%$ & $-0.43 \%$ & $19.88 \%$ & $0 \%$ & $15.85 \%$ \\
\hline
\end{tabular}

eral the $(\ell, S)$ bound more significantly compared to previous methods. These average percentages are calculated by (FL bound - $\ell, S$ bound) $/(\ell, S$ bound). Similar to our previous comparisons, we also provide the average improvements of the Lagrangian relaxation LR2 that relaxes level-linking constraints (i.e., $L R_{2}\left(\mu^{*}\right)$ ) over the FL bound in the column "LR2 vs. FL", calculated by ( $L B\left(L R_{2}\left(\mu^{*}\right)\right)$ - FL bound)/(FL bound). Although one would expect the LR2, the approximation of $L D_{2}$, to improve the FL lower bounds, at first sight this does not seem to be the case for many problem instances, particularly due to negative averages in the LB column of Table 2 . However, as can be seen from the UB column of the table, which indicates $\left(U B\left(L R_{2}\left(\mu^{*}\right)\right)-\mathrm{FL}\right.$ bound $) /(\mathrm{FL}$ bound), these Lagrangian problems are far from optimality, particularly the bigger instances of test sets $\mathrm{C}$ and $\mathrm{D}$, and the challenge here is that these problems need much more time than the assigned default times (or any reasonable amount of time) for optimality or even for an acceptable gap. For testing whether this is the case here, we experimented with a few randomly selected instances from sets $\mathrm{A}+$ and $\mathrm{B}+$ that did not achieve the FL bounds earlier and ran them either until the lower bound was at least as strong as the FL bound or to optimality. For the instances we took for this ad-hoc test, we ended up with bounds that reached at least FL bounds, though we would not be able to generalize this as this was simply for a small subset of the test problems, due to high computational effort. Furthermore, this experiment failed due to memory problems for the few instances from sets $\mathrm{C}$ and $\mathrm{D}$ and hence could not be completed.

Finally, the last two columns of Table 2 should also be addressed briefly. These columns indicate the duality gaps for the two Lagrangian relaxation problems, which can be defined as:

$$
\begin{gathered}
{\left[U B\left(L R_{1}\left(\lambda^{*}\right)\right)-L B\left(L R_{1}\left(\lambda^{*}\right)\right)\right] / L B\left(L R_{1}\left(\lambda^{*}\right)\right)} \\
{\left[U B\left(L R_{2}\left(\mu^{*}\right)\right)-L B\left(L R_{2}\left(\mu^{*}\right)\right)\right] / L B\left(L R_{2}\left(\mu^{*}\right)\right)}
\end{gathered}
$$

Note that these gaps are not related to the original problem and only indicate the problem complexity of these Lagrangian subproblems. As we mentioned before, the LR1 problem is in general comparatively easier to solve than the LR2 problem. We had a total of 11 instances where the LR1 could solve optimally in the assigned default times, compared to none for the LR2. 
Next, we present results for LOTSIZELIB instances in Table 3, where all values are shown explicitly, including the optimal solutions (OPT) in the last column. The table also has a "Heur" column for comparison purposes, which is the lower bound obtained by the heuristic in our companion paper (Akartunalı and Miller [1]), calculated from the first iteration of a relax-and-fix framework. $\mathrm{MC}$ provides significant improvement over the $(\ell, S)$ bound for some of these instances, whereas FL provides negligible improvement over MC. The LR1 is comparatively more efficient on these instances than the LR2. Note that LR1 and LR2 do not necessarily improve MC and FL bounds respectively, similarly to the results for some TDS instances, since these are approximations for $L D_{1}$ and $L D_{2}$. Also, note that all LR2 problems solved optimally for most of the instances, whereas LR1 problems did not finish in quite a few instances after the default time of 180 seconds. This indicates that these instances have the bottleneck not in capacity constraints but in the multi-level structure. This seems to be due in part to the fact that there is a single machine, and the capacity in these problems is comparatively loose.

Table 3 LOTSIZELIB results

\begin{tabular}{|c|c|c|c|c|c|c|c|c|c|}
\hline & \multicolumn{4}{|c|}{ Lower Bounds } & \multicolumn{2}{|c|}{ LR1 (Cap) } & \multicolumn{2}{|c|}{ LR2 (Lev) } & \multirow[b]{2}{*}{ OPT } \\
\hline & $\ell, S$ & $\mathrm{MC}$ & FL & Heur [1] & LB & UB & LB & UB & \\
\hline B & 3,888 & 3,890 & 3,892 & 3,915 & 3,888 & 3,888 & 3,888 & 3,888 & 3,965 \\
\hline $\mathrm{C}$ & 1,904 & 1,993 & 1,998 & 2,067 & 1,904 & 1,904 & 1,904 & 1,905 & 2,083 \\
\hline $\mathrm{D}$ & 4,534 & 4,794 & 4,795 & 4,714 & 4,766 & 6,095 & 4,534 & 4,535 & 6,482 \\
\hline $\mathrm{E}$ & 2,341 & 2,361 & 2,361 & 2,416 & 2,462 & 3,136 & 2,341 & 2,341 & 2,801 \\
\hline $\mathrm{F}$ & 2,075 & 2,098 & 2,111 & 2,099 & 2,237 & 2,459 & 2,079 & 2,079 & 2,429 \\
\hline
\end{tabular}

The detailed results on Multi-LSB instances can be seen in the Tables 510 of "Online Supplement", and the pairwise comparisons are summarized in Table 4, which is organized in the same fashion as Table 2 . The default times for the first two sets are 180 seconds, and for the last two sets 500 seconds. First of all, note that MC improves the $(\ell, S)$ bound poorly in most of the instances. Also note that the LR1 is solved to optimality for all these test problems, and as the table indicates, this approximation of $L D_{1}$ does not often provide an improvement over MC. This might be due in part to poor multipliers generated from the $(\ell, S)$ formulation (also recall that these instances have backlogging variables).

On the other hand, FL improves in general the $(\ell, S)$ bound more significantly than $\mathrm{MC}$, although the improvements are still minuscule. Note that LR2 does not solve to optimality for many test instances, particularly for the hard problems. Similar to the LR1, the LR2 does not provide necessarily an improvement over FL bound, possibly due to poor multipliers. Compared to previous test problems, Multi-LSB instances are parallel to TDS problems, where the bottleneck lies in the capacities rather than the multi-level structure of these problems. 
Table 4 Pairwise comparisons of lower bounds and LR gaps for Multi-LSB instances

\begin{tabular}{|c|c|c|c|c|c|c|c|c|}
\hline \multirow{2}{*}{$\begin{array}{l}\text { Test } \\
\text { Set }\end{array}$} & \multirow{2}{*}{$\begin{array}{c}\mathrm{MC} \text { vs. } \\
\ell, S\end{array}$} & \multicolumn{2}{|c|}{ LR1 vs. MC } & \multirow{2}{*}{$\begin{array}{c}\text { FL vs. } \\
\ell, S\end{array}$} & \multicolumn{2}{|c|}{ LR2 vs. FL } & \multicolumn{2}{|c|}{ LR Gaps } \\
\hline & & $\overline{\mathrm{LB}}$ & UB & & $\overline{\mathrm{LB}}$ & UB & LR1 & LR2 \\
\hline SET1 & $0.02 \%$ & $-0.02 \%$ & $-0.02 \%$ & $0.85 \%$ & $-0.29 \%$ & $-0.28 \%$ & $0.00 \%$ & $0.01 \%$ \\
\hline SET2 & $0.06 \%$ & $-0.06 \%$ & $-0.06 \%$ & $0.28 \%$ & $-0.11 \%$ & $-0.05 \%$ & $0.00 \%$ & $0.06 \%$ \\
\hline SET3 & $6.28 \%$ & $-4.27 \%$ & $-4.27 \%$ & $6.11 \%$ & $-5.14 \%$ & $24.83 \%$ & $0.00 \%$ & $21.92 \%$ \\
\hline SET4 & $1.23 \%$ & $-1.14 \%$ & $-1.14 \%$ & $3.40 \%$ & $-0.99 \%$ & $4.34 \%$ & $0.00 \%$ & $4.76 \%$ \\
\hline
\end{tabular}

\subsection{Summary}

One of our main goals of this paper was to understand the structure of production planning problems and the underlying difficulties that make these problems very hard. In general, the Lagrangian relaxations we tested are helpful for this. First of all, recall that in general the Lagrangian relaxation that relaxes capacity constraints, i.e., $L R 1\left(\lambda^{*}\right)$, provides only slight improvement over the $(\ell, S)$ bound. Also recall that $L R 1\left(\lambda^{*}\right)$ values provide a lower bound to $L D_{1}$. The $L R 1\left(\lambda^{*}\right)$ bound can be seen as an approximation to the convex hull of the uncapacitated problem polyhedron, and our computational results indicate that removing capacities makes the problem much easier. This can also be observed by recalling that the final gaps after the default times were quite small for this Lagrangian relaxation in general.

On the other hand, the facility location reformulation with cover cuts and the Lagrangian relaxation that relaxes the level-linking constraints (although only an approximation to the Lagrangian dual) seem to improve the lower bounds much more significantly. Recall that the cover cuts approximate the intersection of all knapsack sets included in the problem, and $L R 2\left(\mu^{*}\right)$ provides an approximation to the convex hull of the single-level capacitated polyhedrons within the overall multi-level problem. Having higher duality gaps compared to the LR1, this Lagrangian relaxation problem is in general much harder to solve, indicating that the level-linking constraints are not the bottleneck of these problems. A similar comparison is achieved by Jans and Degraeve [21] for single-level problems, where their Lagrangian relaxation relaxing only periodlinking constraints is a harder problem than the one that relaxes capacities. Recall that we did not report computational results on $L D_{3}$, due to the result presented in Corollary 7.

\section{Conclusion}

In this paper, we have provided an extensive survey of different methodologies for obtaining lower bounds for big bucket production planning problems, and presented both theoretical and computational comparisons of them.

In summary, it seems that the multi-level structure by itself makes some of our problems challenging to solve. However, for most instances, and in particular for the most challenging, the single-level, capacitated substructures are 
clearly a much greater contributor to problem difficulty. It is this substructure for which the tools currently at our disposal are evidently not sufficient.

These observations indicate that the main bottleneck with these problems lies in the fact that there is no efficient polyhedral approximation of the multiitem, multi-period, single-level, single-machine capacitated problems. It seems that if we could solve these problems well or even adequately, our ability to solve multi-level bug bucket problems would increase dramatically. While initial efforts to find strong formulations for these problems have been made (e.g. see Miller et al. [32]), this is a fundamental area in which it is crucial for the research community to improve the current state of the art. We will attempt to make contributions in this direction in future research.

Acknowledgements We would like to thank two anonymous referees for their constructive comments that helped us to improve the presentation of the paper.

\section{References}

1. K. Akartunalı and A.J. Miller. A heuristic approach for big bucket multi-level production planning problems. European Journal of Operational Research, 193:396-411, 2009.

2. K. Akartunalı. Computational methods for big bucket production planning problems: Feasible solutions and strong formulations. $\mathrm{PhD}$ thesis, Industrial and Systems Engineering Department, University of Wisconsin-Madison, 2007.

3. S. Anily, M. Tzur and L.A. Wolsey. Multi-item lot-sizing with joint set-up costs. Mathematical Programming, 119(1):79-94, 2009.

4. A. Atamtürk and J.C. Muñoz. A study of the lot-sizing polytope. Mathematical Programming, 98:443-465, 2004

5. I. Barany, T.J. Van Roy, and L.A. Wolsey. Strong formulations for multi-item capacitated lot-sizing. Management Science, 30(10):1255-1261, 1984.

6. I. Barany, T.J. Van Roy, and L.A. Wolsey. Uncapacitated lot sizing: The convex hull of solutions. Mathematical Programming Study, 22:32-43, 1984.

7. G. Belvaux and L.A. Wolsey. bc-prod: A specialized branch-and-cut system for lot-sizing problems. Management Science, 46(5):724-738, 2000.

8. G. Belvaux and L.A. Wolsey. Modelling practical lot-sizing problems as mixed-integer programs. Management Science, 47(7):993-1007, 2001.

9. P.J. Billington, J.O. McClain and L.J. Thomas. Heuristics for multilevel lot-sizingwith a bottleneck. Management Science, 32:989-1006, 1986.

10. G.R. Bitran and H. Matsuo. The multi-item capacitated lot size problem: error bounds of Mannes formulations. Management Science, 32:350-359, 1986.

11. G.R. Bitran and H.H. Yanasse. Computational complexity of the capacitated lot size problem. Management Science, 28(10):1174-1186, 1982.

12. L. Buschkühl, F. Sahling, S. Helber and H. Tempelmeier. Dynamic capacitated lot-sizing problems: a classification and review of solution approaches. OR Spectrum, 32(2):231-261, 2010.

13. M. Constantino. A cutting plane approach to capacitated lot-sizing with start-up costs. Mathematical Programming, 75:353-376, 1996.

14. Z. Degraeve and R. Jans. A new Dantzig-Wolfe reformulation and branch-and-price algorithm for the capacitated lot-sizing problem with setup times. Operations Research, 55(5):909-920, 2007.

15. G.D. Eppen and R.K. Martin. Solving multi-item capacitated lot-sizing problems using variable redefinition. Operations Research, 35(6):832-848, 1987.

16. A. Federgruen and M. Tzur. A simple forward algorithm to solve general dynamic lot sizing models with $n$ periods in $\mathrm{O}(n \log n)$ or $\mathrm{O}(n)$ time. Management Science, 37(8):909$925,1991$. 
17. A. Federgruen, J. Meissner and M. Tzur. Progressive interval heuristics for multi-item capacitated lot sizing problem. Operations Research, 55(3):490-502, 2007.

18. M. Florian and M. Klein. Deterministic production planning with concave costs and capacity constraints. Management Science, 18(1):12-20, 1971.

19. M. Florian, J.K. Lenstra, and H.G. Rinnooy Kan. Deterministic production planning: Algorithms and complexity. Management Science, 26(7):669-679, 1980.

20. O. Günlük and Y. Pochet. Mixing mixed integer inequalities. Mathematical Programming, 90:429-457, 2001.

21. R. Jans and Z. Degraeve. Improved lower bounds for the capacitated lot sizing problem with setup times. Operations Research Letters, 32:185-195, 2004.

22. E. Katok, H.S. Lewis, and T.P. Harrison. Lot sizing in general assembly systems with setup costs, setup times, and multiple constrained resources. Management Science, 44(6):859-877, 1998.

23. J. Krarup and O. Bilde. Plant location, set covering and economic lotsizes: an $O(m n)$ algorithm for structured problems, pages 155-180. Optimierung bei Graphentheoretischen und Ganzzahligen Probleme. Birkhauser Verlag, 1977.

24. S. Küçükyavuz and Y. Pochet. Uncapacitated Lot-Sizing with Backlogging: The Convex Hull. Mathematical Programming, 118(1):151-175, 2009.

25. R. Levi, A. Lodi and M. Sviridenko. Approximation algorithms for the capacitated multi-item lot-sizing problem via flow-cover inequalities. Mathematics of Operations Research, 33(2):461-474, 2008.

26. M. Loparic, Y. Pochet, and L.A. Wolsey. The uncapacitated lot-sizing problem with sales and safety stocks. Mathematical Programming, 89:487-504, 2001.

27. LOTSIZELIB. Lot-sizing problems: A library of models and matrices. http://www.core.ucl.ac.be/wolsey/lotsizel.htm, 1999.

28. A.S. Manne. Programming of economic lot sizes. Management Science, 4(2):115135, 1958.

29. H. Marchand and L.A. Wolsey. The 0-1 knapsack problem with a single continuous variable. Mathematical Programming, 85(1):15-33, 1999.

30. H. Marchand and L.A. Wolsey. Aggregation and mixed integer rounding to solve mips. Operations Research, 49(3):363-371, 2001.

31. A.J. Miller. Polyhedral Approaches to Capacitated Lot-Sizing Problems. PhD thesis, Industrial and Systems Engineering Department, Georgia Institute of Technology, 1999.

32. A.J. Miller, G.L. Nemhauser, and M.W.P. Savelsbergh. Solving the multi-item capacitated lot-sizing problem with setup times by branch-and-cut. CORE Discussion Paper 2000/39, CORE, UCL, Belgium, 2000.

33. A.J. Miller, G.L. Nemhauser, and M.W.P. Savelsbergh. On the polyhedral structure of a multi-item production planning model with setup times. Mathematical Programming, 94:375-405, 2003

34. Multi-LSB. Multi-item lot-sizing problems with backlogging: A library of test instances. Available at http://personal.strath.ac.uk/kerem.akartunali/research/multi-lsb/, 2010.

35. Y. Pochet and L.A. Wolsey. Lot-size models with backlogging: Strong reformulations and cutting planes. Mathematical Programming, 40:317-335, 1988.

36. Y. Pochet and L.A. Wolsey. Solving multi-item lot-sizing problems using strong cutting planes. Management Science, 37(1):53-67, 1991.

37. Y. Pochet and L.A. Wolsey. Polyhedra for lot-sizing with Wagner-Whitin costs. Mathematical Programming, 67:297-323, 1994

38. Y. Pochet and L.A. Wolsey. Production Planning by Mixed Integer Programming. Springer, 2006.

39. R.L. Rardin and L.A. Wolsey. Valid inequalities and projecting the multicommodity extended formulation for uncapacitated fixed charge network flow problems. European Journal of Operational Research, 71:95-109, 1993.

40. J.-P.P. Richard, I.R. de Farias, and G.L. Nemhauser. Lifted inequalities for 0-1 mixed integer programming: Basic theory and algorithms. Mathematical Programming, 98(13):89-113, 2003

41. J.-P.P. Richard, I.R. de Farias, and G.L. Nemhauser. Lifted inequalities for 0-1 mixed integer programming: Superlinear lifting. Mathematical Programming, 98(1-3):115-143, 2003. 
42. N.C. Simpson and S.S. Erenguc. Modeling multiple stage manufacturing systems with generalized costs and capacity issues. Naval Research Logistics, 52:560-570, 2005.

43. H. Stadtler. Multilevel lot sizing with setup times and multiple constrained resources: Internally rolling schedules with lot-sizing windows. Operations Research, 51:487-502, 2003.

44. H. Tempelmeier and M. Derstroff. A lagrangean-based heuristic for dynamic multilevel multiitem constrained lotsizing with setup times. Management Science, 42(5):738-757, 1996.

45. J.M. Thizy and L.N. Van Wassenhove. Lagrangean relaxation for the multi-item capacitated lot-sizing problem: A heuristic implementation. IIE Transactions, 17(4):308-313, 1985.

46. W.W. Trigeiro, L.J. Thomas, and J.O. McClain. Capacitated lot sizing with setup times. Management Science, 35:353-366, 1989.

47. M. Van Vyve and Y. Pochet. A general heuristic for production planning problems. INFORMS Journal of Computing, 16(3):316-327, 2004.

48. H.M. Wagner and T.M. Whitin. Dynamic version of the economic lot size model. Management Science, 5:89-96, 1958.

49. L.A. Wolsey. Integer Programming. Wiley-Interscience, 1998.

50. L.A. Wolsey. Solving multi-item lot-sizing problems with an MIP solver using classification and reformulation. Management Science, 48(12):1587-1602, 2002.

51. W.I. Zangwill. A backlogging model and a multi-echelon model of a dynamic economic lot size production system-a network approach. Management Science, 15(9):506-527, 1969.

\section{APPENDIX: Detailed Results (Online Supplement)}

This section is prepared to present all detailed computational results that are too overwhelming for and therefore only summarized in the paper titled "A Computational Analysis of Lower Bounds for Big Bucket Production Planning Problems". It is aimed that this level of detail can help other researchers to get better insight, as well as have benchmark values when needed.

All the tables are structured the same way, as following: The first column indicates the specific name of the instance. The next four columns present the lower bound values obtained, in the order of $(\ell, S)$ inequalities (root node solution of the Branch\&Bound tree), multi-commodity (MC) reformulation (without the effect of any solver cuts), facility location (FL) reformulation (with all cover cuts generated by the solver), and lower bound obtained by our heuristic (see reference [1] in the paper). Then, the next two columns provide the lower and upper bounds of the 1st Lagrangian problem (relaxing capacity), followed by the two columns presenting the lower and upper bounds of the 2nd Lagrangian problem (relaxing level). Finally, the last column indicates the best solution we are aware of. Note that for the Lagrangian relaxations, we use the dual optimal values of the constraints from the strong LP relaxation as multipliers, and we set default times of 180 seconds for A+, B+, SET1 and SET2 instances, and 500 seconds for C, D, SET3, SET4 instances. Note that if the Lagrangian relaxation subproblem is not solved to optimality in this preassigned time, the lower and upper bounds of this Lagrangian subproblem provide us the range where the actual lower bound of the Lagrangian relaxation lies. 
Table 5 TDS Instances Detailed Computational Results, set A+

\begin{tabular}{|c|c|c|c|c|c|c|c|c|c|}
\hline & \multicolumn{4}{|c|}{ Lower Bounds } & \multicolumn{2}{|c|}{ LR1 (Cap) } & \multicolumn{2}{|c|}{ LR2 (Lev) } & \multirow{2}{*}{$\begin{array}{l}\text { Best } \\
\text { Soln }\end{array}$} \\
\hline & $\ell, S$ & $\mathrm{MC}$ & $\mathrm{FL}$ & Heuristic & LB & UB & $\overline{\mathrm{LB}}$ & UB & \\
\hline AG501130 & 116,183 & 116,600 & 118,340 & 119,146 & 117,808 & 123,203 & 120,764 & 127,683 & 153,418 \\
\hline AG501131 & 107,829 & 108,106 & 108,987 & 109,714 & 109,298 & 115,656 & 108,822 & 117,533 & 145,225 \\
\hline AG501132 & 118,677 & 118,957 & 119,986 & 121,740 & 120,163 & 123,663 & 120,454 & 128,249 & 154,191 \\
\hline AG501141 & 133,424 & 134,008 & 135,519 & 134,421 & 135,078 & 141,548 & 136,547 & 147,696 & 171,895 \\
\hline AG501142 & 145,508 & 145,873 & 147,646 & 148,911 & 146,527 & 151,197 & 149,002 & 156,488 & 192,582 \\
\hline AG502130 & 122,353 & 123,904 & 125,925 & 128,101 & 125,087 & 125,472 & 127,119 & 134,118 & 167,927 \\
\hline AG502131 & 109,085 & 109,501 & 110,500 & 111,001 & 111,043 & 116,443 & 109,959 & 121,005 & 145,322 \\
\hline AG502141 & 134,971 & 135,527 & 136,973 & 136,353 & 136,792 & 141,900 & 139,060 & 146,767 & 173,640 \\
\hline AG502232 & 97,032 & 97,488 & 97,890 & 97,632 & 98,529 & 101,859 & 98,206 & 102,415 & 121,108 \\
\hline AG502531 & 102,340 & 103,252 & 102,817 & 103,506 & 103,216 & 105,542 & 103,211 & 109,727 & 129,080 \\
\hline AK501131 & 96,968 & 96,983 & 99,966 & 99,020 & 97,892 & 98,030 & 97,811 & 112,060 & 123,366 \\
\hline AK501132 & 101,699 & 101,781 & 103,276 & 103,077 & 102,289 & 102,887 & 102,847 & 109,206 & 123,473 \\
\hline AK501141 & 134,805 & 134,943 & 139,399 & 136,428 & 135,487 & 136,315 & 137,303 & 163,011 & 170,897 \\
\hline AK501142 & 134,880 & 135,006 & 138,151 & 135,875 & 135,122 & 137,204 & 137,867 & 151,661 & 161,262 \\
\hline AK501432 & 92,533 & 92,605 & 92,968 & 93,546 & 94,679 & 94,679 & 93,270 & 93,645 & 109,249 \\
\hline AK502130 & 102,222 & 102,245 & 106,358 & 103,949 & 103,054 & 103,460 & 104,351 & 117,191 & 127,889 \\
\hline AK502131 & 93,369 & 93,423 & 95,912 & 94,969 & 93,778 & 94,145 & 94,338 & 101,804 & 115,819 \\
\hline AK502132 & 96,312 & 96,396 & 98,423 & 97,233 & 96,933 & 97,092 & 97,644 & 104,528 & 118,319 \\
\hline AK502142 & 127,792 & 127,977 & 129,654 & 129,034 & 128,226 & 130,758 & 129,863 & 138,752 & 146,616 \\
\hline AK502432 & 88,980 & 89,088 & 89,550 & 89,609 & 90,193 & 91,779 & 89,995 & 91,225 & 105,415 \\
\hline
\end{tabular}

Table 6 TDS Instances Detailed Computational Results, set B+

\begin{tabular}{|c|c|c|c|c|c|c|c|c|c|}
\hline & \multicolumn{4}{|c|}{ Lower Bounds } & \multicolumn{2}{|c|}{ LR1 (Cap) } & \multicolumn{2}{|c|}{ LR2 (Lev) } & \multirow{2}{*}{$\begin{array}{l}\text { Best } \\
\text { Soln }\end{array}$} \\
\hline & $\ell, S$ & $\mathrm{MC}$ & FL & Heuristic & $\mathrm{LB}$ & UB & $\mathrm{LB}$ & UB & \\
\hline BG511132 & 108,772 & 109,045 & 109,875 & 110,466 & 110,136 & 114,629 & 109,545 & 116,781 & 137,637 \\
\hline BG511142 & 133,158 & 133,652 & 134,424 & 133,880 & 134,500 & 137,991 & 134,648 & 146,913 & 159,769 \\
\hline BG512131 & 104,054 & 104,483 & 105,158 & 105,804 & 105,469 & 110,855 & 104,580 & 112,766 & 138,752 \\
\hline BG512132 & 114,786 & 115,314 & 115,894 & 116,135 & 115,931 & 119,395 & 115,156 & 125,132 & 151,770 \\
\hline BG512142 & 142,917 & 143,659 & 144,840 & 143,848 & 144,161 & 148,340 & 145,305 & 158,261 & 199,051 \\
\hline BG521132 & 108,324 & 108,559 & 109,338 & 110,024 & 109,805 & 113,609 & 109,109 & 115,077 & 138,133 \\
\hline BG521142 & 131,363 & 131,908 & 132,996 & 132,604 & 132,905 & 137,629 & 133,224 & 141,350 & 156,694 \\
\hline BG522130 & 113,540 & 114,876 & 116,472 & 121,578 & 115,240 & 119,850 & 115,961 & 123,968 & 154,581 \\
\hline BG522132 & 113,382 & 113,838 & 114,305 & 115,158 & 114,551 & 119,158 & 114,262 & 121,255 & 147,894 \\
\hline BG522142 & 137,126 & 137,782 & 138,608 & 138,077 & 138,405 & 142,417 & 138,851 & 144,180 & 186,268 \\
\hline BK511131 & 92,602 & 92,640 & 93,964 & 94,411 & 93,107 & 94,310 & 93,304 & 99,779 & 120,303 \\
\hline BK511132 & 95,323 & 95,355 & 97,283 & 95,938 & 95,942 & 96,844 & 96,310 & 103,668 & 115,416 \\
\hline BK511141 & 125,307 & 125,494 & 126,753 & 126,769 & 125,679 & 127,256 & 126,534 & 135,597 & 162,629 \\
\hline BK512131 & 90,733 & 90,787 & 92,253 & 92,058 & 91,391 & 92,036 & 91,568 & 96,009 & 113,536 \\
\hline BK512132 & 90,814 & 90,858 & 92,896 & 91,346 & 91,738 & 92,208 & 91,870 & 98,554 & 112,809 \\
\hline BK521131 & 92,350 & 92,382 & 93,469 & 94,164 & 92,881 & 94,004 & 92,884 & 97,318 & 118,217 \\
\hline BK521132 & 94,257 & 94,317 & 96,197 & 94,957 & 94,932 & 95,914 & 95,110 & 101,441 & 117,423 \\
\hline BK521142 & 124,988 & 125,257 & 126,384 & 125,480 & 125,333 & 128,448 & 126,548 & 134,871 & 153,805 \\
\hline BK522131 & 90,532 & 90,588 & 91,731 & 91,742 & 91,131 & 91,802 & 91,291 & 96,184 & 111,339 \\
\hline BK522142 & 119,559 & 119,739 & 120,794 & 119,625 & 120,047 & 124,160 & 120,956 & 127,283 & 148,471 \\
\hline
\end{tabular}


Table 7 TDS Instances Detailed Computational Results, set C

\begin{tabular}{|c|c|c|c|c|c|c|c|c|c|}
\hline & \multicolumn{4}{|c|}{ Lower Bounds } & \multicolumn{2}{|c|}{ LR1 (Cap) } & \multicolumn{2}{|c|}{ LR2 (Lev) } & \multirow{2}{*}{$\begin{array}{l}\text { Best } \\
\text { Soln }\end{array}$} \\
\hline & $\ell, S$ & $\mathrm{MC}$ & FL & Heuristic & LB & UB & $\mathrm{LB}$ & UB & \\
\hline CG501120 & $1,011,260$ & $1,012,042$ & $1,025,118$ & $1,027,177$ & $1,012,992$ & $1,022,396$ & $1,017,258$ & $1,109,345$ & $1,252,308$ \\
\hline CG501131 & 472,421 & 472,711 & 475,464 & 478,437 & 473,125 & 476,392 & 472,947 & 513,188 & 614,303 \\
\hline CG501141 & 627,035 & 627,631 & 630,113 & 628,114 & 628,641 & 631,308 & 627,980 & 678,899 & 777,831 \\
\hline CG501121 & 945,696 & 946,442 & 953,112 & 959,756 & 948,052 & 953,730 & 946,612 & $1,045,688$ & $1,247,493$ \\
\hline CG502221 & 724,648 & 725,517 & 725,827 & 728,105 & 726,515 & 743,421 & 724,779 & 765,713 & 889,548 \\
\hline CG501132 & 561,827 & 562,158 & 566,137 & 606,568 & 562,887 & 567,636 & 567,379 & 597,061 & 842,734 \\
\hline CG501222 & 697,129 & 698,410 & 699,934 & 699,021 & 699,024 & 718,231 & 697,860 & 723,508 & 858,289 \\
\hline CG501142 & 754,238 & 757,449 & 761,826 & 824,887 & 757,128 & 758,835 & 764,794 & 802,021 & $1,146,638$ \\
\hline CG501122 & $1,161,383$ & $1,162,216$ & $1,171,502$ & $1,281,687$ & $1,165,839$ & $1,178,726$ & $1,174,289$ & $1,243,710$ & $1,787,833$ \\
\hline CG502222 & 704,096 & 705,161 & 707,153 & 708,597 & 706,766 & 725,192 & 704,971 & 753,284 & 873,858 \\
\hline CK501120 & 141,900 & 142,034 & 143,869 & 143,260 & 142,581 & 145,659 & 143,212 & 156,264 & 176,187 \\
\hline CK501221 & 101,028 & 101,108 & 101,570 & 101,105 & 101,299 & 103,024 & 101,114 & 106,030 & 123,066 \\
\hline CK501121 & 131,993 & 132,185 & 133,494 & 132,840 & 132,708 & 137,522 & 132,496 & 147,865 & 169,804 \\
\hline CK502221 & 101,478 & 101,740 & 102,242 & 101,899 & 101,968 & 103,730 & 101,623 & 107,423 & 122,596 \\
\hline CK501222 & 97,937 & 98,050 & 98,858 & 98,096 & 98,313 & 100,271 & 98,267 & 102,163 & 122,485 \\
\hline CK501422 & 101,864 & 102,007 & 102,660 & 102,150 & 102,135 & 102,981 & 103,846 & 107,102 & 124,315 \\
\hline CK502222 & 98,052 & 98,236 & 98,898 & 98,282 & 98,450 & 100,835 & 98,333 & 104,359 & 119,965 \\
\hline CK501122 & 153,861 & 154,358 & 156,048 & 155,485 & 154,841 & 155,914 & 155,016 & 165,574 & 206,646 \\
\hline CK501132 & 75,257 & 75,301 & 76,198 & 75,782 & 75,648 & 76,311 & 75,780 & 80,388 & 98,248 \\
\hline CK501142 & 90,218 & 90,347 & 91,277 & 90,673 & 90,477 & 91,215 & 90,701 & 96,230 & 115,918 \\
\hline
\end{tabular}

Table 8 TDS Instances Detailed Computational Results, set D

\begin{tabular}{|c|c|c|c|c|c|c|c|c|c|}
\hline & \multicolumn{4}{|c|}{ Lower Bounds } & \multicolumn{2}{|c|}{ LR1 (Cap) } & \multicolumn{2}{|c|}{ LR2 (Lev) } & \multirow{2}{*}{$\begin{array}{l}\text { Best } \\
\text { Soln }\end{array}$} \\
\hline & $\ell, S$ & $\mathrm{MC}$ & FL & Heuristic & LB & UB & LB & UB & \\
\hline DG512141 & 609,464 & 610,630 & 611,291 & 615,992 & 610,613 & 610,613 & 609,599 & 659,071 & 736,181 \\
\hline DG512131 & 465,272 & 466,156 & 466,203 & 469,460 & 466,333 & 466,333 & 465,372 & 495,481 & 581,932 \\
\hline DG012132 & 554,595 & 556,651 & 559,610 & 555,689 & 556,441 & 556,441 & 554,922 & 781,344 & $3,160,347$ \\
\hline DG012142 & 756,588 & 758,120 & 763,304 & 756,588 & 757,387 & 757,387 & 756,898 & $1,001,177$ & $3,121,762$ \\
\hline DG012532 & 554,167 & 555,261 & 556,877 & 555,032 & 555,045 & 555,045 & 554,167 & 775,666 & $1,194,004$ \\
\hline DG012542 & 756,062 & 756,956 & 759,793 & 756,062 & 756,563 & 756,563 & 756,159 & 982,363 & $1,413,476$ \\
\hline DG512132 & 512,330 & 513,440 & 514,386 & 514,682 & 512,722 & 512,722 & 512,376 & 554,333 & $2,909,628$ \\
\hline DG512142 & 678,733 & 679,821 & 681,450 & 682,205 & 679,062 & 679,062 & 678,777 & 854,902 & $3,583,354$ \\
\hline DG512532 & 509,567 & 511,041 & 510,510 & 512,147 & 510,670 & 510,670 & 509,587 & 542,328 & 584,491 \\
\hline DG512542 & 674,241 & 675,180 & 675,969 & 677,189 & 674,734 & 674,734 & 674,241 & 715,533 & 767,428 \\
\hline
\end{tabular}


Table 9 Multi-LSB Detailed Computational Results, SET1 instances 1-20

\begin{tabular}{|c|c|c|c|c|c|c|c|c|c|}
\hline & \multicolumn{4}{|c|}{ Lower Bounds } & \multicolumn{2}{|c|}{ LR1 (Cap) } & \multicolumn{2}{|c|}{ LR2 (Lev) } & \multirow{2}{*}{$\begin{array}{l}\text { Best } \\
\text { Soln }\end{array}$} \\
\hline & $\ell, S$ & $\mathrm{MC}$ & $\overline{F L}$ & Heuristic & LB & UB & $\mathrm{LB}$ & UB & \\
\hline SET1_01 & 17,888 & 17,888 & 18,173 & 18,840 & 17,888 & 17,888 & 17,888 & 17,972 & 22,781 \\
\hline SET1_02 & 23,534 & 23,534 & 23,656 & 24,134 & 23,534 & 23,534 & 23,534 & 23,534 & 28,624 \\
\hline SET1_03 & 21,227 & 21,227 & 21,346 & 21,676 & 21,227 & 21,227 & 21,227 & 21,227 & 26,349 \\
\hline SET1_04 & 22,232 & 22,232 & 22,334 & 23,175 & 22,232 & 22,232 & 22,232 & 22,232 & 26,337 \\
\hline SET1_05 & 21,446 & 21,446 & 21,540 & 21,994 & 21,446 & 21,446 & 21,446 & 21,446 & 25,621 \\
\hline SET1_06 & 22,974 & 22,974 & 23,072 & 23,636 & 22,974 & 22,974 & 22,974 & 22,974 & 26,741 \\
\hline SET1_07 & 20,360 & 20,360 & 20,386 & 21,125 & 20,360 & 20,360 & 20,360 & 20,360 & 24,693 \\
\hline SET1_08 & 25,582 & 25,582 & 25,616 & 26,249 & 25,582 & 25,582 & 25,582 & 25,582 & 29,810 \\
\hline SET1_09 & 16,321 & 16,321 & 16,442 & 17,013 & 16,321 & 16,321 & 16,321 & 16,338 & 21,146 \\
\hline SET1_10 & 17,998 & 17,998 & 18,151 & 18,945 & 17,998 & 17,998 & 17,998 & 18,011 & 22,863 \\
\hline SET1_11 & 11,080 & 11,080 & 11,237 & 11,407 & 11,080 & 11,080 & 11,164 & 11,169 & 12,956 \\
\hline SET1_12 & 24,721 & 24,721 & 24,762 & 25,238 & 24,721 & 24,721 & 24,721 & 24,725 & 26,985 \\
\hline SET1_13 & 20,782 & 20,788 & 20,830 & 21,195 & 20,782 & 20,782 & 20,782 & 20,786 & 23,129 \\
\hline SET1_14 & 22,264 & 22,268 & 22,331 & 22,745 & 22,264 & 22,264 & 22,264 & 22,264 & 25,720 \\
\hline SET1_15 & 12,401 & 12,404 & 12,805 & 12,575 & 12,401 & 12,401 & 12,564 & 12,564 & 14,121 \\
\hline SET1_16 & 15,122 & 15,122 & 15,356 & 15,387 & 15,122 & 15,122 & 15,543 & 15,543 & 17,542 \\
\hline SET1_17 & 20,468 & 20,475 & 20,498 & 20,864 & 20,468 & 20,468 & 20,468 & 20,468 & 23,404 \\
\hline SET1_18 & 11,075 & 11,077 & 11,366 & 11,456 & 11,075 & 11,075 & 11,462 & 11,462 & 12,300 \\
\hline SET1_19 & 13,276 & 13,276 & 13,528 & 13,342 & 13,276 & 13,276 & 13,388 & 13,388 & 17,448 \\
\hline SET1_20 & 14,101 & 14,101 & 14,177 & 14,612 & 14,101 & 14,101 & 14,101 & 14,113 & 17,167 \\
\hline
\end{tabular}

Table 10 Multi-LSB Detailed Computational Results, SET1 instances 21-30 and SET2 instances 1-10

\begin{tabular}{|c|c|c|c|c|c|c|c|c|c|}
\hline & \multicolumn{4}{|c|}{ Lower Bounds } & \multicolumn{2}{|c|}{ LR1 (Cap) } & \multicolumn{2}{|c|}{ LR2 (Lev) } & \multirow{2}{*}{$\begin{array}{l}\text { Best } \\
\text { Soln }\end{array}$} \\
\hline & $\ell, S$ & $\mathrm{MC}$ & FL & Heuristic & LB & UB & LB & UB & \\
\hline SET1_21 & 10,159 & 10,166 & 10,429 & 10,392 & 10,159 & 10,159 & 10,325 & 10,325 & 12,421 \\
\hline SET1_22 & 38,040 & 38,056 & 38,166 & 38,040 & 38,040 & 38,040 & 38,040 & 38,077 & 40,158 \\
\hline SET1_23 & 29,331 & 29,343 & 29,376 & 29,355 & 29,331 & 29,331 & 29,331 & 29,331 & 30,606 \\
\hline SET1_24 & 28,858 & 28,858 & 29,074 & 29,250 & 28,858 & 28,858 & 28,886 & 28,886 & 32,174 \\
\hline SET1_25 & 51,371 & 51,371 & 51,403 & 51,371 & 51,371 & 51,371 & 51,371 & 51,371 & 53,009 \\
\hline SET1_26 & 39,379 & 39,379 & 39,463 & 39,488 & 39,379 & 39,379 & 39,402 & 39,402 & 41,442 \\
\hline SET1_27 & 40,838 & 40,838 & 40,838 & 40,918 & 40,838 & 40,838 & 40,838 & 40,838 & 43,320 \\
\hline SET1_28 & 39,846 & 39,864 & 39,894 & 40,144 & 39,846 & 39,846 & 39,857 & 39,857 & 40,993 \\
\hline SET1_29 & 23,155 & 23,165 & 23,275 & 23,232 & 23,155 & 23,155 & 23,182 & 23,182 & 25,606 \\
\hline SET1_30 & 68,989 & 68,989 & 69,074 & 68,989 & 68,989 & 68,989 & 68,989 & 68,989 & 70,868 \\
\hline SET2_01 & 46,116 & 46,116 & 46,207 & 46,591 & 46,116 & 46,116 & 46,116 & 46,116 & 55,039 \\
\hline SET2_02 & 47,780 & 47,780 & 47,861 & 48,159 & 47,780 & 47,780 & 47,780 & 47,780 & 57,825 \\
\hline SET2_03 & 40,551 & 40,551 & 40,610 & 40,814 & 40,551 & 40,551 & 40,551 & 40,551 & 49,147 \\
\hline SET2_04 & 36,347 & 36,347 & 36,564 & 36,808 & 36,347 & 36,347 & 36,347 & 36,430 & 44,656 \\
\hline SET2_05 & 45,395 & 45,395 & 45,508 & 45,784 & 45,395 & 45,395 & 45,395 & 45,395 & 55,650 \\
\hline SET2_06 & 45,902 & 45,902 & 45,939 & 45,902 & 45,902 & 45,902 & 45,902 & 45,902 & 54,361 \\
\hline SET2_07 & 52,825 & 52,825 & 52,939 & 53,108 & 52,825 & 52,825 & 52,825 & 52,825 & 61,140 \\
\hline SET2_08 & 48,033 & 48,033 & 48,280 & 48,632 & 48,033 & 48,033 & 48,084 & 48,084 & 56,444 \\
\hline SET2_09 & 37,553 & 37,553 & 37,661 & 37,943 & 37,553 & 37,553 & 37,553 & 37,553 & 44,523 \\
\hline SET2_10 & 38,751 & 38,751 & 38,898 & 39,181 & 38,751 & 38,751 & 38,751 & 38,751 & 49,481 \\
\hline
\end{tabular}


Table 11 Multi-LSB Detailed Computational Results, SET2 instances 11-30

\begin{tabular}{|c|c|c|c|c|c|c|c|c|c|}
\hline & \multicolumn{4}{|c|}{ Lower Bounds } & \multicolumn{2}{|c|}{ LR1 (Cap) } & \multicolumn{2}{|c|}{ LR2 (Lev) } & \multirow{2}{*}{$\begin{array}{l}\text { Best } \\
\text { Soln }\end{array}$} \\
\hline & $\ell, S$ & $\mathrm{MC}$ & $\overline{F L}$ & Heuristic & LB & UB & $\mathrm{LB}$ & $\overline{\mathrm{UB}}$ & \\
\hline SET2_11 & 65,210 & 65,211 & 65,213 & 65,648 & 65,210 & 65,210 & 65,210 & 65,210 & 69,177 \\
\hline SET2_12 & 62,792 & 62,792 & 62,979 & 62,792 & 62,792 & 62,792 & 62,803 & 62,803 & 66,914 \\
\hline SET2_13 & 34,778 & 34,778 & 34,882 & 34,987 & 34,778 & 34,778 & 34,885 & 34,885 & 40,114 \\
\hline SET2_14 & 62,907 & 62,907 & 62,993 & 62,907 & 62,907 & 62,907 & 62,907 & 62,916 & 67,201 \\
\hline SET2_15 & 59,079 & 59,079 & 59,125 & 59,079 & 59,079 & 59,079 & 59,079 & 59,079 & 61,616 \\
\hline SET2_16 & 75,682 & 75,682 & 75,698 & 75,682 & 75,682 & 75,682 & 75,682 & 75,682 & 79,576 \\
\hline SET2_17 & 36,809 & 36,818 & 36,918 & 36,925 & 36,809 & 36,809 & 36,826 & 36,935 & 41,484 \\
\hline SET2_18 & 77,873 & 77,874 & 77,935 & 78,087 & 77,873 & 77,873 & 77,873 & 77,873 & 83,200 \\
\hline SET2_19 & 54,981 & 54,981 & 55,120 & 55,484 & 54,981 & 54,981 & 55,026 & 55,026 & 59,010 \\
\hline SET2_20 & 119,568 & 119,568 & 119,588 & 119,568 & 119,568 & 119,568 & 119,568 & 119,568 & 122,974 \\
\hline SET2_21 & 22,281 & 22,315 & 22,557 & 22,281 & 22,281 & 22,281 & 22,643 & 22,643 & 24,459 \\
\hline SET2_22 & 51,279 & 51,279 & 51,439 & 51,279 & 51,279 & 51,279 & 51,414 & 51,414 & 53,690 \\
\hline SET2_23 & 29,793 & 30,067 & 30,210 & 29,793 & 29,793 & 29,793 & 29,814 & 29,815 & 33,969 \\
\hline SET2_24 & 65,891 & 65,891 & 65,984 & 65,891 & 65,891 & 65,891 & 65,891 & 65,891 & 68,727 \\
\hline SET2_25 & 75,627 & 75,628 & 75,745 & 75,627 & 75,627 & 75,627 & 75,705 & 75,705 & 78,266 \\
\hline SET2_26 & 60,952 & 61,002 & 61,173 & 60,977 & 60,952 & 60,952 & 60,988 & 60,988 & 63,558 \\
\hline SET2_27 & 53,016 & 53,016 & 53,052 & 53,016 & 53,016 & 53,016 & 53,016 & 53,441 & 54,797 \\
\hline SET2_28 & 44,545 & 44,552 & 44,705 & 44,549 & 44,545 & 44,545 & 44,923 & 44,923 & 46,733 \\
\hline SET2_29 & 93,631 & 93,638 & 93,659 & 93,631 & 93,631 & 93,631 & 93,632 & 93,632 & 96,281 \\
\hline SET2_30 & 68,324 & 68,333 & 68,573 & 68,573 & 68,324 & 68,324 & 68,324 & 68,324 & 71,919 \\
\hline
\end{tabular}

Table 12 Multi-LSB Detailed Computational Results, SET3 instances 1-20

\begin{tabular}{|c|c|c|c|c|c|c|c|c|c|}
\hline & \multicolumn{4}{|c|}{ Lower Bounds } & \multicolumn{2}{|c|}{ LR1 (Cap) } & \multicolumn{2}{|c|}{ LR2 (Lev) } & \multirow{2}{*}{$\begin{array}{l}\text { Best } \\
\text { Soln }\end{array}$} \\
\hline & $\ell, S$ & $\mathrm{MC}$ & FL & Heuristic & LB & UB & $\mathrm{LB}$ & UB & \\
\hline SET3_01 & 65,668 & 71,594 & 71,584 & 71,533 & 66,984 & 66,984 & 65,761 & 112,652 & 209,129 \\
\hline SET3_02 & 82,342 & 89,855 & 89,887 & 89,980 & 84,865 & 84,865 & 82,704 & 105,740 & 243,511 \\
\hline SET3_03 & 74,209 & 82,398 & 82,440 & 81,340 & 77,086 & 77,086 & 74,611 & 99,483 & 235,198 \\
\hline SET3_04 & 78,282 & 85,258 & 85,229 & 86,280 & 80,716 & 80,716 & 78,436 & 108,664 & 240,339 \\
\hline SET3_05 & 76,607 & 83,692 & 83,667 & 84,430 & 78,931 & 78,931 & 76,884 & 102,852 & 227,758 \\
\hline SET3_06 & 79,093 & 88,689 & 88,737 & 85,674 & 82,910 & 82,910 & 79,625 & 112,534 & 235,642 \\
\hline SET3_07 & 72,979 & 79,067 & 79,181 & 79,668 & 75,365 & 75,365 & 73,098 & 105,466 & 237,218 \\
\hline SET3_08 & 88,610 & 94,504 & 94,481 & 98,469 & 92,108 & 92,108 & 89,213 & 129,505 & 251,628 \\
\hline SET3_09 & 64,180 & 67,768 & 67,760 & 73,019 & 64,336 & 64,336 & 64,180 & 85,114 & 216,025 \\
\hline SET3_10 & 66,878 & 74,333 & 74,324 & 73,902 & 67,928 & 67,928 & 66,912 & 92,540 & 229,242 \\
\hline SET3_11 & 42,946 & 46,063 & 45,997 & 47,273 & 43,902 & 43,902 & 43,012 & 69,501 & 152,962 \\
\hline SET3_12 & 86,047 & 95,953 & 95,980 & 97,672 & 90,412 & 90,412 & 87,641 & 112,402 & 217,497 \\
\hline SET3_13 & 74,643 & 81,477 & 81,348 & 83,699 & 75,379 & 75,379 & 74,987 & 102,771 & 224,670 \\
\hline SET3_14 & 85,209 & 91,252 & 91,435 & 94,426 & 86,813 & 86,813 & 85,493 & 102,438 & 225,657 \\
\hline SET3_15 & 40,715 & 43,551 & 43,343 & 45,265 & 40,843 & 40,843 & 40,750 & 74,085 & 167,494 \\
\hline SET3_16 & 46,548 & 50,868 & 50,784 & 51,811 & 48,528 & 48,528 & 48,360 & 62,509 & 162,616 \\
\hline SET3_17 & 71,555 & 78,132 & 77,988 & 82,199 & 72,458 & 72,458 & 71,837 & 95,764 & 212,399 \\
\hline SET3_18 & 39,533 & 40,406 & 40,259 & 46,743 & 39,658 & 39,658 & 39,616 & 57,199 & 112,468 \\
\hline SET3_19 & 47,495 & 50,636 & 50,497 & 53,815 & 48,266 & 48,266 & 47,636 & 84,711 & 154,981 \\
\hline SET3_20 & 58,189 & 60,240 & 60,125 & 62,614 & 58,529 & 58,529 & 59,753 & 95,852 & 191,639 \\
\hline
\end{tabular}


Table 13 Multi-LSB Detailed Computational Results, SET3 instances 21-30 and SET4 instances 1-10

\begin{tabular}{|c|c|c|c|c|c|c|c|c|c|}
\hline & \multicolumn{4}{|c|}{ Lower Bounds } & \multicolumn{2}{|c|}{ LR1 (Cap) } & \multicolumn{2}{|c|}{ LR2 (Lev) } & \multirow{2}{*}{$\begin{array}{l}\text { Best } \\
\text { Soln }\end{array}$} \\
\hline & $\ell, S$ & $\mathrm{MC}$ & FL & Heuristic & LB & UB & $\mathrm{LB}$ & UB & \\
\hline SET3_21 & 44,182 & 45,435 & 45,383 & 53,138 & 44,359 & 44,359 & 44,182 & 60,262 & 150,758 \\
\hline SET3_22 & 130,235 & 138,607 & 138,279 & 136,582 & 133,995 & 133,995 & 130,930 & 142,716 & 292,199 \\
\hline SET3_23 & 96,810 & 102,993 & 102,912 & 107,981 & 99,719 & 99,719 & 96,939 & 115,205 & 240,643 \\
\hline SET3_24 & 105,300 & 110,117 & 109,994 & 115,086 & 105,327 & 105,327 & 105,300 & 136,353 & 292,996 \\
\hline SET3_25 & 203,044 & 210,031 & 209,928 & 210,037 & 204,955 & 204,955 & 203,044 & 212,110 & 349,975 \\
\hline SET3_26 & 145,184 & 152,864 & 152,545 & 160,639 & 146,938 & 146,938 & 145,198 & 155,347 & 323,870 \\
\hline SET3_27 & 145,420 & 154,121 & 153,805 & 154,499 & 148,698 & 148,698 & 145,674 & 169,988 & 343,486 \\
\hline SET3_28 & 145,227 & 153,083 & 153,327 & 152,942 & 147,940 & 147,940 & 145,927 & 162,729 & 254,008 \\
\hline SET3_29 & 79,813 & 87,043 & 86,551 & 84,552 & 81,494 & 81,494 & 80,206 & 96,912 & 207,127 \\
\hline SET3_30 & 274,018 & 283,252 & 282,958 & 275,167 & 276,810 & 276,810 & 274,018 & 284,338 & 431,136 \\
\hline SET4_01 & 16,353 & 16,532 & 18,093 & 21,961 & 16,353 & 16,353 & 16,951 & 23,694 & 58,720 \\
\hline SET4_02 & 31,541 & 32,773 & 34,074 & 41,393 & 31,541 & 31,541 & 31,726 & 33,919 & 82,496 \\
\hline SET4_03 & 24,864 & 25,616 & 27,464 & 33,058 & 24,864 & 24,864 & 24,864 & 28,061 & 73,740 \\
\hline SET4_04 & 27,786 & 28,837 & 30,023 & 36,512 & 27,786 & 27,786 & 27,928 & 31,426 & 73,651 \\
\hline SET4_05 & 25,450 & 26,353 & 27,335 & 35,022 & 25,450 & 25,450 & 25,450 & 29,755 & 67,874 \\
\hline SET4_06 & 30,632 & 31,495 & 32,990 & 40,513 & 30,632 & 30,632 & 31,054 & 35,402 & 79,781 \\
\hline SET4_07 & 22,650 & 23,189 & 24,599 & 31,952 & 22,650 & 22,650 & 23,884 & 30,365 & 65,736 \\
\hline SET4_08 & 40,532 & 42,512 & 43,131 & 48,381 & 40,532 & 40,532 & 40,538 & 41,812 & 88,388 \\
\hline SET4_09 & 13,490 & 13,557 & 14,687 & 21,182 & 13,490 & 13,490 & 14,650 & 19,585 & 57,070 \\
\hline SET4_10 & 15,542 & 15,553 & 16,857 & 25,595 & 15,542 & 15,542 & 16,041 & 26,902 & 59,319 \\
\hline
\end{tabular}

Table 14 Multi-LSB Detailed Computational Results, SET4 instances 11-30

\begin{tabular}{|c|c|c|c|c|c|c|c|c|c|}
\hline & \multicolumn{4}{|c|}{ Lower Bounds } & \multicolumn{2}{|c|}{ LR1 (Cap) } & \multicolumn{2}{|c|}{ LR2 (Lev) } & \multirow{2}{*}{$\begin{array}{l}\text { Best } \\
\text { Soln }\end{array}$} \\
\hline & $\ell, S$ & $\mathrm{MC}$ & $\overline{\mathrm{FL}}$ & Heuristic & $\mathrm{LB}$ & UB & $\overline{\mathrm{LB}}$ & UB & \\
\hline SET4_11 & 12,802 & 12,996 & 13,825 & 17,303 & 12,802 & 12,802 & 13,675 & 15,205 & 28,989 \\
\hline SET4_12 & 43,341 & 44,527 & 45,100 & 50,868 & 43,341 & 43,341 & 44,523 & 46,502 & 78,062 \\
\hline SET4_13 & 28,152 & 28,736 & 30,049 & 34,945 & 28,152 & 28,152 & 28,152 & 33,352 & 53,833 \\
\hline SET4_14 & 56,174 & 57,052 & 57,302 & 64,255 & 56,174 & 56,174 & 56,406 & 57,049 & 82,406 \\
\hline SET4_15 & 14,628 & 14,715 & 15,304 & 15,863 & 14,628 & 14,628 & 15,244 & 16,260 & 26,980 \\
\hline SET4_16 & 17,171 & 17,529 & 17,990 & 22,405 & 17,172 & 17,172 & 17,662 & 19,874 & 35,280 \\
\hline SET4_17 & 29,001 & 29,886 & 30,581 & 36,480 & 29,225 & 29,225 & 29,237 & 31,729 & 54,515 \\
\hline SET4_18 & 19,184 & 19,213 & 19,309 & 22,584 & 19,185 & 19,185 & 19,705 & 19,997 & 26,279 \\
\hline SET4_19 & 10,724 & 10,769 & 11,780 & 14,950 & 10,724 & 10,724 & 12,581 & 15,411 & 31,974 \\
\hline SET4_20 & 18,718 & 18,858 & 19,702 & 23,969 & 18,731 & 18,731 & 19,420 & 21,014 & 39,983 \\
\hline SET4_21 & 15,812 & 16,243 & 16,819 & 18,259 & 15,812 & 15,812 & 16,386 & 17,720 & 25,899 \\
\hline SET4_22 & 91,715 & 93,010 & 93,185 & 93,869 & 91,733 & 91,733 & 92,228 & 92,310 & 120,166 \\
\hline SET4_23 & 55,058 & 55,601 & 56,077 & 57,298 & 55,151 & 55,151 & 55,562 & 56,132 & 76,857 \\
\hline SET4_24 & 58,919 & 59,231 & 59,512 & 63,700 & 58,919 & 58,919 & 59,213 & 60,947 & 85,119 \\
\hline SET4_25 & 171,987 & 172,779 & 172,904 & 173,663 & 171,987 & 171,987 & 171,987 & 171,988 & 201,717 \\
\hline SET4_26 & 110,570 & 111,393 & 111,703 & 117,746 & 110,570 & 110,570 & 110,570 & 110,577 & 142,090 \\
\hline SET4_27 & 101,114 & 102,197 & 102,182 & 103,873 & 101,471 & 101,471 & 101,267 & 101,340 & 139,874 \\
\hline SET4_28 & 112,892 & 113,353 & 114,022 & 113,987 & 112,892 & 112,892 & 112,987 & 112,987 & 126,027 \\
\hline SET4_29 & 51,149 & 51,394 & 51,776 & 56,304 & 51,149 & 51,149 & 51,253 & 51,253 & 68,320 \\
\hline SET4_30 & 241,678 & 243,702 & 243,998 & 242,481 & 241,801 & 241,801 & 241,678 & 241,693 & 267,976 \\
\hline
\end{tabular}

